\title{
Temporal Assessment of the Medicinal Plant Trade in Public Markets of the State of Paraíba, Northeastern Brazil
}

\section{Ezequiel da Costa Ferreira}

Universidade Federal da Paraiba

Reinaldo Farias Paiva de Lucena

Universidade Federal da Paraiba

\section{Rainer W. Bussmann}

Institute of Botany and Bakuriani Alpine Botanical Garden

Narel Y. Paniagua-Zambrana

Institute of Botany and Bakuriani Alpine Botanical Garden

Denise Dias da Cruz ( $\nabla$ denidcruz@dse.ufpb.br)

Universidade Federal da Paraiba https://orcid.org/0000-0001-6840-5432

\section{Research Article}

Keywords: Urban ethnobotany, Caatinga, Atlantic Forest, similarity analysis, relative importance

Posted Date: May 27th, 2021

DOl: https://doi.org/10.21203/rs.3.rs-557959/v1

License: (9) This work is licensed under a Creative Commons Attribution 4.0 International License.

Read Full License 


\section{Temporal assessment of the medicinal plant trade in public markets of the state of Paraíba, northeastern Brazil}

Ezequiel da Costa Ferreira ${ }^{1,2^{*}}$, Reinado Farias Paiva de Lucena ${ }^{2,3}$, Rainer W. Bussmann ${ }^{4}$, Narel Y. Paniagua-Zambrana ${ }^{4}$, Denise Dias da Cruz ${ }^{1,2 *}$

*Correspondence: ezequielcostaf@gmail.com; denidcruz@dse.ufpb.br

${ }^{1}$ Laboratório de Ecologia Terrestre, Dept. de Sistemática e Ecologia, Centro de Ciências

Exatas e da Natureza, Universidade Federal da Paraíba, João Pessoa, PB, Brazil, 58051-900.

e-mail: denidcruz@dse.ufpb.br

${ }^{2}$ Programa de Pós-Graduação em Desenvolvimento e Meio Ambiente, PRODEMA,

Universidade Federal da Paraíba, João Pessoa, PB, Brazil, 58051-900.

${ }^{3}$ Instituto de Biociências, Universidade Federal do Mato Grosso do Sul, Campo Grande, MS, Brazil, 79070-900.

E-mail: reinaldolucena.dr@gmail.com

${ }^{4}$ Department of Ethnobotany, Institute of Botany and Bakuriani Alpine Botanical Garden; Ilia State University; 1 Botanical Str.; 0105 Tbilisi; Georgia

E-mail: rainer.bussmann@iliauni.edu.ge

E-mail: narel.paniagua@iliauni.edu.ge 


\section{ABSTRACT}

Background: Open and public markets are the main providers of medicinal plants in urban environments. The present study evaluated the medicinal plants sold in public markets in different municipalities in the mesoregions of the state of Paraíba, northeast of Brazil, and the possible variations in the supply of these plants in the markets over the course of a year.

Methods: Thirty-five traders of medicinal plants were interviewed in four mesoregions of different climatic and phytophysiognomic characteristics (ranging from Caatinga to Atlantic Forest). The versatility of the species sold was elucidated using the Relative Importance Index (IR), and the set of species sold by each informant in each mesoregion was compared with each other by similarity analysis Anosim-One, and by the analysis of main coordinates.

Results: Punica granatum, Zingiber officinale, and Myracrodruon urundeuva were the species with the highest RI. The analysis of similarity showed distinct differences between the Sertão and all other mesoregions. The analysis highlighted that a mesoregion had an intermediate similarity pattern in relation to the others, for which we suggest the term "biocultural ecotone" or "cultotone". The absence of 88 species in at least one of the trading locations at some stage of the fieldwork was recorded.

Conclusions: The commercialized species do not seem to have a presence / absence relationship in relation to the period of the year or the mesoregion, and there seem to have been changes in the inventory of plants commercialized in markets in recent years. We identified an intermediate zone of knowledge and use of species commercialized between the studied localities.

Keywords: Urban ethnobotany, Caatinga, Atlantic Forest, similarity analysis, relative importance. 


\section{Background}

The knowledge about, and use of medicinal plants, are themes that remain one ofthe main study topics in ethnobotany. Many recent studies in Brazil and around the globe have recorded the knowledge and use of medicinal plants, both in rural [e.g. 1-8] and urban areas [e.g. 9-15].

In urban areas, open and public markets are some of the main sources of medicinal plants. There it is possible to find these products traded, and to observe variations with regard to both the plant parts sold and the inventory of available species over time. Several studies observed the predominance of the medicinal use of non-permanent plant structures, such as leaves $[8,16,17]$. Some studies in Brazil showed that overall, changes in the list of traded species occured as a function of the temporal availability or the demand for certyain species in the market $[10,18-20]$. In other cases, it was possible to observe a relatively constant inventory of medicinal plants, with the inclusion of some new species over time, e.g. in La Paz, Bolivia [21], both in the short and medium term [21]. When observing broader temporal contexts, the changes in the inventory of medicinal plants can become more evident, as observed in Peru, where the local pharmacopeia has been changing since the colonial period [22].

The climate and the predominant phytophysiognomy in a given region can also influence the set of traded species or the plant parts used. From this perspective, the more ample use of permanent plant structures such as bark and roots, has been often documented from drier areas, such as the Caatinga [23-25] (semi-arid region of Brazil), as well as in Savannah and Desert areas in Africa [26-28]. In humid areas, it has been more common to observe a greater use of leaves, such as in the Atlantic Forest [29] and the Amazon [30], as well as in rainforest areas in Asia [17,31,32].

In addition to the availability of the specific plant parts used, several other factors can influence the availability of traded products, such as seasonality [18,20], demand [18], and 
local environmental factors [33]. Repeated sampling can be useful to identify and understand these variations in the medicinal plant products available for trade $[10,33]$.

Variations in the availability of traded plants have also been observed when comparing different public markets, even in nearby areas [21] or in long-term comparisons with previous studies [19,21]. Another issue that can influence the trade and use of medicinal plants is globalization, especially due to the increase and use of social and digital media to advertise and trade these products [34], and the medicinal plant trade in the international context [13].

The present study aimed to assess the medicinal plants traded in the public markets of municipalities in four mesoregions of the state of Paraíba, northeastern Brazil, each with its independent climatic and phytophysiognomy characteristics, but with two well-defined biomes: the Caatinga semi-arid region, and the Atlantic Forest humid region. The assessment was performed by documenting the species used, and the possible variations in their availability in the markets throughout the year. We hypothesized that there would be a greater influence of seasonality on the availability of medicinal plants for trade in the interior of the state, a region with a semi-arid climate, than in the coast, which is subject to higher humidity over the year, especially with respect to native species.

In addition, based on the analysis of the information recorded in our study, we propose the possible existence of a zone that we called "biocultural ecotone" or "cultotone", i.e. a region marked not only by a transition in the vegetation but perhaps of the knowledge and practice in the use of medicinal plants, somewhat similar to the concept of ecotone. This perspective could be an interesting guiding tool for future research involving the trade in medicinal plants. 


\section{Material and methods}

\section{Study area}

The study was conducted in public markets of seven municipalities of Paraíba, distributed in the four mesoregions of the state: João Pessoa and Sapé (Mata Mesoregion), Guarabira and Solânea (Agreste Mesoregion), Monteiro (Borborema Mesoregion), and Patos and Itaporanga (Sertão Mesoregion). The predominant phytophysiognomy in the municipalities of João Pessoa and Sapé is Atlantic Forest, while Caatinga vegetation predominates in the remaining municipalities (Figure 1). These mesoregions are defined by socioeconomic and environmental characteristics, showing marked climatic variations. In 2017, when our fieldwork was already in progress, the IBGE (Brazilian Institute of Geography and Statistics) changed the division of the geographic regions of Brazil, modifying the configuration and the classification as Mesoregions and Microregions into Intermediate Regions and Immediate Regions, respectively [35]. For this study, we adopted the Mesoregion and Microregion classification, because this classification allows better visualization of the different phytophysiognomies of the state, considering that the new Regional Division of Brazil is more focused on socioeconomic aspects [35], not highlighting the environmental differences.

The Mata Paraibana Mesoregion is characterized by a hot and humid climate [36] with three climate types according to the classification by Köppen: Aw (tropical, with a dry season in winter), Am (high rainfall), and As (hot and humid tropical, with dry winter) [37]. The Mata Paraibana shelters what little is left of the Atlantic Forest in Paraíba, most which has been destroyed anthropogenic impact, especially the expansion of sugarcane production. This zone also incorporates beaches, plateaus, floodplains, and estuaries [36].

The Agreste comprises a transition area between the humid and the semi-arid climate [36], belonging to the Köppen climate types As and Bsh (hot semi-arid) [37]. Its vegetation represents also a transition area between the Atlantic Forest and the Caatinga [36]. 
The Borborema comprises the central area of the state of Paraíba, located in the geomorphological unit of the Borborema Plateau [36]. It shows the Köppen climate types As and Bsh [37], with the lowest rainfall levels in the state, and salty, thin, and rocky soils.

The Sertão comprises several depressions, with a semi-arid climate, and a vegetation characteristic of the Caatinga [36]. This area is divided between the Köppen climate types Bsh (hot semi-arid) and As (hot and humid tropical, with dry winter) [37].

Before beginning the interviews, previous visits were made to identify locations that met the objectives of the study. It was impossible to conduct the research in some of the visited municipalities (Figure 1), either because they did not have a public market or because there were no medicinal plants traded in the market.

\section{Data collection}

Visits were made to the trading locations of medicinal plants in the public markets of the municipalities. After initial contact to properly present and explain the purposes of the study, the medicinal plant traders in the markets were invited to sign the Free Consent Form (TCLE) required by the National Health Council through the Research Ethics Committee (Resolution 466/12) (Protocol: 82943618.0.0000.5188) and to participate in the research. A total of 35 traders were interviewed (13 in the Mata Paraibana; $10 \mathrm{n}$ in the Agreste; 4 in the Borborema; 8 in the Sertão). The free list technique was used based on the following question: "Which medicinal plants do you sell?" Subsequently, for each plant, details on their origin (was a plant from the local vegetation?; did it come from other regions?; was it imported?), considering both native and exotic species, and registering their applications, properties, preparation, parts used, and contraindications. Repeat interviews were performed each trimester during one year, strating August 2017, in order to evaluate if a species was either absent or added in relation to the previous periods. During each interview, a list of all plants 
available in a specific trade location was compiled, and further details were obtained on the use of each species.

The identification of the traded species was made by acquiring fertile specimens in the markets and collecting the cited species in the field when possible, and then comparing the material with the corresponding literature. The names and families of the species were confirmed using REFLORA (Flora do Brasil 2020) [38] and the Missouri Botanical Garden database (Tropicos) [39]. The herborized plants were sent to the Herbarium Jaime Coelho de Moraes (EAN) of the Federal University of Paraíba (UFPB), Center of Agricultural Sciences (CCA) for confirmation of the identification and incorporation into the plant collection

\section{Data analysis}

The therapeutic indications mentioned by the informants were classified into body systems (categories defined by the WHO for each property) [40]. The NSC (Number of Body Systems) and NP (Number of Properties) for each species were calculated according to Bennett and Prance [41], with the following equations:

\section{$\mathrm{NSC}=\mathrm{NSCE} / \mathrm{NSCEV}$}

Where NSC refers to the number of body systems, resulting from the division of the number of body systems treated by a given species (NSCE) by the total number of body systems treated by the most versatile species (NSCEV), considering as the most versatile the species that obtained the greatest diversity of body systems.

The following equation was used for the NP:

$$
\mathrm{NP}=\mathrm{NPE} / \mathrm{NPEV} \text {. }
$$

Where NP is the number of properties, resulting from the division of the number of properties attributed to a given species (NPE) by the number of properties attributed to the 
most versatile species (NPEV), considering as the most versatile the species that obtained the highest number of properties.

Subsequently, also based on Bennett and Prance [41], the Relative Importance (RI) of each species was calculated by the following equation:

$$
\mathrm{RI}=\mathrm{NSC} / \mathrm{NP}
$$

This method highlights the most versatile species or those with the greatest diversity of uses. It consists of a quantitative method that is not directly influenced by the number of citations for a given species but rather by the diversity of applications inferred to a species. The maximum value obtained is 2 ; the closer to this value is the RI of the plant, the greater its versatility, also considering that the RI of the species is high when $\geq 1$.

The one-way ANOSIM permutation test was used to assess the degree of similarity of the species used between mesoregions (Bray-Curtis distance and 9999 permutations). This test produces an $\mathrm{R}$ result that ranges from -1 to +1 , which may indicate no significant difference between groups $(\mathrm{R}<0.25)$, while values between $0.25<\mathrm{R}<0.5$ indicate some data similarity and values of $\mathrm{R}>0.75$ indicate different results, with total difference when $\mathrm{R}=1$. Principal Coordinates Analysis (PCoA) using the Bray-Curtis distance was employed to generate a graph representing these differences between the cited species. The software Past 3.22 was used in the analyses. Data tabulation in the software was made based on a presence and absence matrix in binary code, in which 1 represented the presence of the species considered in the trade location and 0 represented the absence.

\section{Results}

A total of 163 species were identified at least to genus level, belonging to 151 genera and 76 families. Seventeen species remained unidentified. The most common families were Fabaceae (19 species), Asteraceae (12), Lamiaceae (11), and Myrtaceae (6) (Table 1). A high 
relative importance value was recorded $(\mathrm{RI} \geq 1)$ for 32 species, among which 11 were native to Brazil, and 21 were exotic (Table 2). The species that obtained the highest RI values were Punica granatum L. (Pomegranate; RI = 2), Zingiber officinale Roscoe (Ginger; 1.78), and Myracrodruon urundeuva Allemão (Aroeira; 1.69) (Tables 1 and 2). The categories with the highest number of citations for each mesoregion were: unspecified diseases and symptoms; digestive system; endocrine system, nutrition, and metabolism; and respiratory system, with varying prevalence according to each mesoregion (Figure 2).

The plant parts traded most commonly for medicinal use were leaves, bark, and seeds, varying only with regard to their prevalence in each mesoregion (Figure 3). The use of flowers stood out in the Borborema region (Figure 3). In the context of this study the stem was considered as both the shoot and its subterraneous structures (rhizome and bulb), when present.

Among the recorded species, 27 (16.07\% of the total) were found only in the Mata mesoregion. However, the Sertão showed the highest exclusivity, with 45 species $(26.79 \%)$. The Agreste mesoregion had only four exclusive species (2.38\%), while the Borborema mesoregion had only three exclusive species (1.79\%) (Table 1). The one-way ANOSIM multivariate analysis demonstrated similarity among the set of species traded in the mesoregions, with exception for the Sertão, which was significantly different from allo other mesoregions $(\mathrm{R}=0.2136 ; \mathrm{p}<0.0018)$, showing significant variation compared to the Mata $(\mathrm{R}=0.2632 ; \mathrm{p}<0.0074)$, Agreste $(\mathrm{R}=0.3752 ; \mathrm{p}<0.0036)$, and Borborema mesoregions $(\mathrm{R}=0.3888 ; \mathrm{p}<0.0187)$. The principal coordinate analysis $(\mathrm{PCoA})$ highlighted the similarity between the plant species traded by the informants in three of the mesoregions comopared to the Sertão (Figure 4). 
Table 1. Medicinal plants traded in public markets of the Mata, Agreste, Borborema, and Sertão mesoregions of Paraíba, northeastern Brasil,

2 indications, and categories of medicinal use and Relative Importance (RI) of each species. Categories: BCS = Blood and cardiovascular system;

$3 \quad$ CUD $=$ Cultural diseases; DIS = Digestive system; ENM = Endocrine system, nutrition, and metabolism; EXI = External injuries; INT =

4 Intoxication; $\mathrm{MBD}=$ Mental and behavioral diseases; MSS = Musculoskeletal system; NEO = Neoplasms; NES = Nervous system; PAD =

5 Parasitic diseases; PRP = Pregnancy and parturition; RES = Respiratory system; RSS = Reproductive system and sexual health; SES = Sensory

6 system; SST = Skin and subcutaneous tissue; UDS = Unspecified diseases and symptoms; URS = Urinary system.

\begin{tabular}{llll}
\hline Family & Local/Vernacular & Medicinal use categories & Mesoregion \\
Scientific name & name & & RI \\
\hline Acanthaceae & & RES (cough, hoarseness, expectoration); & Mata \\
Justicia pectoralis Jacq. & Chachambá & DIS (indigestion) & 0.47
\end{tabular}

\begin{tabular}{lllr}
\hline Adoxaceae & & & \\
& & NES (tranquilizer), UDS (fever), BCS (high blood pressure), PAD & Mata; Agreste; \\
Sambucus australis Cham. \& & Sabugueira & (measles, infection), RES (cough, flu) & Sertão \\
Schltdl. & & & 0.81 \\
\end{tabular}

Alismataceae 


\section{Amaranthaceae}

Dysphania ambrosioides (L.)

Mosyakin \& Clemants

\section{Amaryllidaceae}

Allium sativum $\mathrm{L}$.

Alho

Allium sp.
Cebolinha branca
ENM (cholesterol, "reduce the levels", weight loss); RSS (erectile

dysfunction); BCS (anticoagulant, stroke); PAD (worm infection); RES Mata; Sertão (tiredness)

Mata; Agreste;

RES (cough, flu, hoarseness, expectoration, bronchitis, sinusitis, common

Borborema;

Sertão

\section{Anacardiaceae}

SST (wound healing); UDS (inflammation);

Mata; Agreste;

Anacardium occidentale L. Cajú roxo 


\begin{abstract}
Myracrodruon
urundeuva

Allemão
\end{abstract}

Schinus terebinthifolia Raddi

Aroeira

Schnopsis brasiliensis Engl. Baraúna

Annona muricata L.

Graviola

Xylopia aromática (Lam.) Mart. Imbira

\section{Annonaceae}

UDS (inflammation); URS (prostate); SST (wound healing, pruritus); DIS (gastritis); EXI (stop bleeding); MSS (bone inflammation); PAD (antibiotic); ENM (menopause); BCS (high blood pressure, blood)

Mata; Agreste;

Sertão

UDS (inflammation); SST (wound healing); DIS (intestine inflammation) Mata

UDS (inflammation); SST (wound healing); URS (kidneys); MSS Mata; Agreste;

(spine); RSS (prostate)

Sertão

DIS (gastritis); NEO (cancer); ENM ("reduce the levels" diabetes); UDS

(inflammation)

Mata; Sertão

DIS (bellyache, indigestion, stomach, stomach ache); UDS (pain); RSS Mata; Agreste;

(menstrual cramps); MSS (back pain)

Sertão

\section{Apiaceae}

NES (tranquilizer, insomnia); UDS (pain); BCS (heart, jaundice, high Mata; Agreste;

Anethum graveolens $\mathrm{L} . \quad$ Endro

blood pressure, tachycardia); DIS (intestinal colic, stomach ache, Borborema;

gallbladder pain, constipation, indigestion); RSS (menstrual cramps) Sertão

Centella sp.

Centelha asiática

ENM (weight loss)

Mata; Sertão

Coriandrum sativum $\mathrm{L}$.

Coentro

BCS (high blood pressure); ENM (cholesterol, menopause); DIS Mata; Agreste; 
(indigestion, throat problems); UDS (dizziness, headache); SES Borborema;

(labyrinthitis)

Sertão

NES (tranquilizer, depression, insomnia); RES (sinusitis, runny nose); Mata; Agreste;

Foeniculum vulgare Mill. $\quad$ Erva doce

BCS (high blood pressure, heart); UDS (fever, dizziness, headache); INT Borborema;

(Intoxication); ENM (menopause); DIS (bellyache)

Sertão

NES (tranquilizer, depression, insomnia); RES (sinusitis, runny nose); Mata; Agreste;

Pimpinella anisum $\mathrm{L}$

Erva doce

BCS (high blood pressure, heart); UDS (fever, dizziness, headache); INT Borborema;

(Intoxication); ENM (menopause); DIS (bellyache)

Sertão

\begin{tabular}{|c|c|c|c|c|}
\hline \multicolumn{5}{|l|}{ Apocynaceae } \\
\hline Hancornia speciosa Gomes & Mangaba & BCS (high blood pressure) & Mata & 0.17 \\
\hline \multicolumn{5}{|l|}{ Aquifoliaceae } \\
\hline Ilex sp. & Chá mate & DIS (indigestion) & Mata; Borborema & 0.17 \\
\hline \multicolumn{5}{|l|}{ Arecaceae } \\
\hline Cocos nucifera $\mathrm{L}$. & Côco & BCS (jaundice); URS (diuretic) & Sertão & 0.35 \\
\hline Copernicia prunifera (Mill.) & Carnaúba & URS (kidneys) & Sertão & 0.17 \\
\hline
\end{tabular}

H.E. Moore

MSS (spine); URS (kidneys, kidney stones, urinary tract infection); DIS Mata; Agreste; 


\begin{tabular}{|c|c|c|c|c|}
\hline \multicolumn{5}{|l|}{ Aristolochiaceae } \\
\hline Aristolochia sp. & $\begin{array}{l}\text { Cipó-de-mil- } \\
\text { homem }\end{array}$ & DIS (indigestion) & Mata & 0.17 \\
\hline \multicolumn{5}{|l|}{ Asparagaceae } \\
\hline Agave sp. & Agave branco & UDS (inflammation); ENM (cholesterol) & Agreste; Sertão & 0.35 \\
\hline Sansevieria trifasciata Prain & $\begin{array}{l}\text { Espada de São } \\
\text { Jorge }\end{array}$ & URS (kidneys); UDS (inflammation) & Sertão & 0.35 \\
\hline \multicolumn{5}{|l|}{ Asphodelaceae } \\
\hline Aloe vera (L.) Burm. f. & Babosa & SST (wound healing) & Mata & 0.17 \\
\hline \multicolumn{5}{|l|}{ Asteraceae } \\
\hline Acanthospermum hispidum DC. & Espinho de cigano & RES (flu, bronchitis) & Sertão & 0.24 \\
\hline Ageratum conyzoides $\mathrm{L}$. & Mentraste & RSS (regulation of menstruation); UDS (inflammation) & Sertão & 0.35 \\
\hline Artemisia sp. & Artemísia & UDS (inflammation) & Mata & 0.17 \\
\hline Baccharis sp. & Carqueja & $\begin{array}{l}\text { ENM (liver, liver fat, weight loss, diabetes, cholesterol, blood fat); URS } \\
\text { (diuretic, kidneys) }\end{array}$ & $\begin{array}{l}\text { Mata; Agreste; } \\
\text { Borborema; } \\
\text { Sertão }\end{array}$ & 0.72 \\
\hline
\end{tabular}




\begin{tabular}{|c|c|c|c|c|}
\hline Bidens pilosa $\mathrm{L}$. & Picão preto & UDS (inflammation) & Mata & 0.17 \\
\hline Egletes viscosa (L.) Less. & Macela & $\begin{array}{l}\text { DIS (indigestion, diarrhea, stomach, liver, intestine, intestinal infection); } \\
\text { ENM (diabetes) }\end{array}$ & $\begin{array}{l}\text { Mata; Agreste; } \\
\text { Borborema; } \\
\text { Sertão }\end{array}$ & 0.66 \\
\hline $\begin{array}{l}\text { Gymnanthemum amygdalinum } \\
\text { (Delile) Sch. Bip. ex Walp. }\end{array}$ & Alcachofra & ENM (cholesterol, diabetes, liver inflammation, liver fat) & $\begin{array}{l}\text { Mata; Borborema; } \\
\text { Sertão }\end{array}$ & 0.36 \\
\hline Helianthus annuus $\mathrm{L}$. & Girassol & $\begin{array}{l}\text { UDS (dizziness); SES (labyrinthitis); BCS (thrombosis, CVA); } \\
\text { musculoskeletal system (rheumatism, bursitis); BCS (high blood } \\
\text { pressure) }\end{array}$ & $\begin{array}{l}\text { Mata; Agreste; } \\
\text { Borborema; } \\
\text { Sertão }\end{array}$ & 0.99 \\
\hline Matricaria chamomilla $\mathrm{L}$. & Camomila & $\begin{array}{l}\text { NES (tranquilizer, depression, insomnia); UDS (fever); NEO (cancer); } \\
\text { URS (urethral inflammation); SST (lighten skin spots); BCS (high blood } \\
\text { pressure) }\end{array}$ & $\begin{array}{l}\text { Mata; Agreste; } \\
\text { Borborema; } \\
\text { Sertão }\end{array}$ & 1.17 \\
\hline Solidago chilensis Meyen & Arnica & UDS (pain); MSS (joints); EXI (wounds, blood clot) & Mata; Sertão & 0.58 \\
\hline $\begin{array}{l}\text { Taraxacum officinale F.H. } \\
\text { Wigg. }\end{array}$ & Dente de leão & $\begin{array}{l}\text { ENM (liver fat, weight loss); NES (memory); MSS (joints); DIS } \\
\text { (intestine); BCS (blood circulation) }\end{array}$ & $\begin{array}{l}\text { Mata; Borborema; } \\
\text { Sertão }\end{array}$ & 0.93 \\
\hline Vernonanthura phosphoric & Assa peixe & URS (kidneys) & Mata & 0.17 \\
\hline
\end{tabular}




\begin{tabular}{|c|c|c|c|c|}
\hline \multicolumn{5}{|l|}{ Bignoniaceae } \\
\hline Anemopaegma sp. & Catuaba & UDS (inflammation); RSS (aphrodisiac); MSS (bone pain) & Mata; Sertão & 0.52 \\
\hline $\begin{array}{l}\text { Handroanthus heptaphyllus } \\
\text { (Vell.) Mattos }\end{array}$ & Pau d'arco roxo & $\begin{array}{l}\text { UDS (pain, inflammation); NEO (cancer); RSS (cysts, myoma, ovary } \\
\text { problems); MSS (rheumatism) }\end{array}$ & Mata; Sertão & 0.88 \\
\hline Handroanthus sp. & Pau d'arco & URS (kidneys) & Agreste & 0.17 \\
\hline Jacaranda sp. & Caroba & SST (pruritus, skin irritation); BCS (depurative) & Sertão & 0.41 \\
\hline \multicolumn{5}{|l|}{ Bixaceae } \\
\hline Bixa orellana $\mathrm{L}$. & Urucum & ENM (reduce cholesterol) & Mata; Sertão & 0.17 \\
\hline \multicolumn{5}{|l|}{ Boraginaceae } \\
\hline Heliotropium indicum $\mathrm{L}$. & Fedegoso & PRP ("female cleaning after childbirth") & Sertão & 0.17 \\
\hline $\begin{array}{l}\text { Heliotropium nicotianaefolium } \\
\text { Poir }\end{array}$ & Sete Sangrias & URS (kidneys) & Mata; Sertão & 0.17 \\
\hline Symphytum officinale L. & Confrei & ENM (uric acid); SES (labyrinthitis); UDS (inflammation) & Mata; Sertão & 0.52 \\
\hline Varronia curassavica Jacq. & Erva balieira & UDS (inflammation) & Mata & 0.17 \\
\hline
\end{tabular}

\section{Brassicaceae}

Brassica sp.

Mostarda

BCS (thrombosis, CVA, stroke, circulation); MSS (rheumatism); UDS Mata; Agreste;

Borborema; 
Rorippa nasturtium-aquaticum

(L.) Hayek

Agrião

RES (bronchitis, asthma)

Sertão

Burseraceae

Commiphora leptophloeos

Imburana

RES (flu), MSS (osteoarthritis)

Mata; Agreste;

(Mart.) J.B. Gillett

\section{Cactaceae}

Cereus jamacaru DC.

Cardeiro

URS (kidneys)

Sertão

Melocactus zehntneri (Britton \&

Rose) Luetzelb.

Coroa de Frade

RES (tiredness, asthma)

Mata

\section{Capparaceae}

Cynophalla flexuosa

(L.) J

Presl

Feijão brabo MSS (back pain)

Sertão

\section{Caprifoliacea}

Valeriana sp.

Valeriana

NES (tranquilizer)

Mata

\section{Caryocaraceae}

Caryocar sp

Pequi

RES (flu, cough)

Sertão 


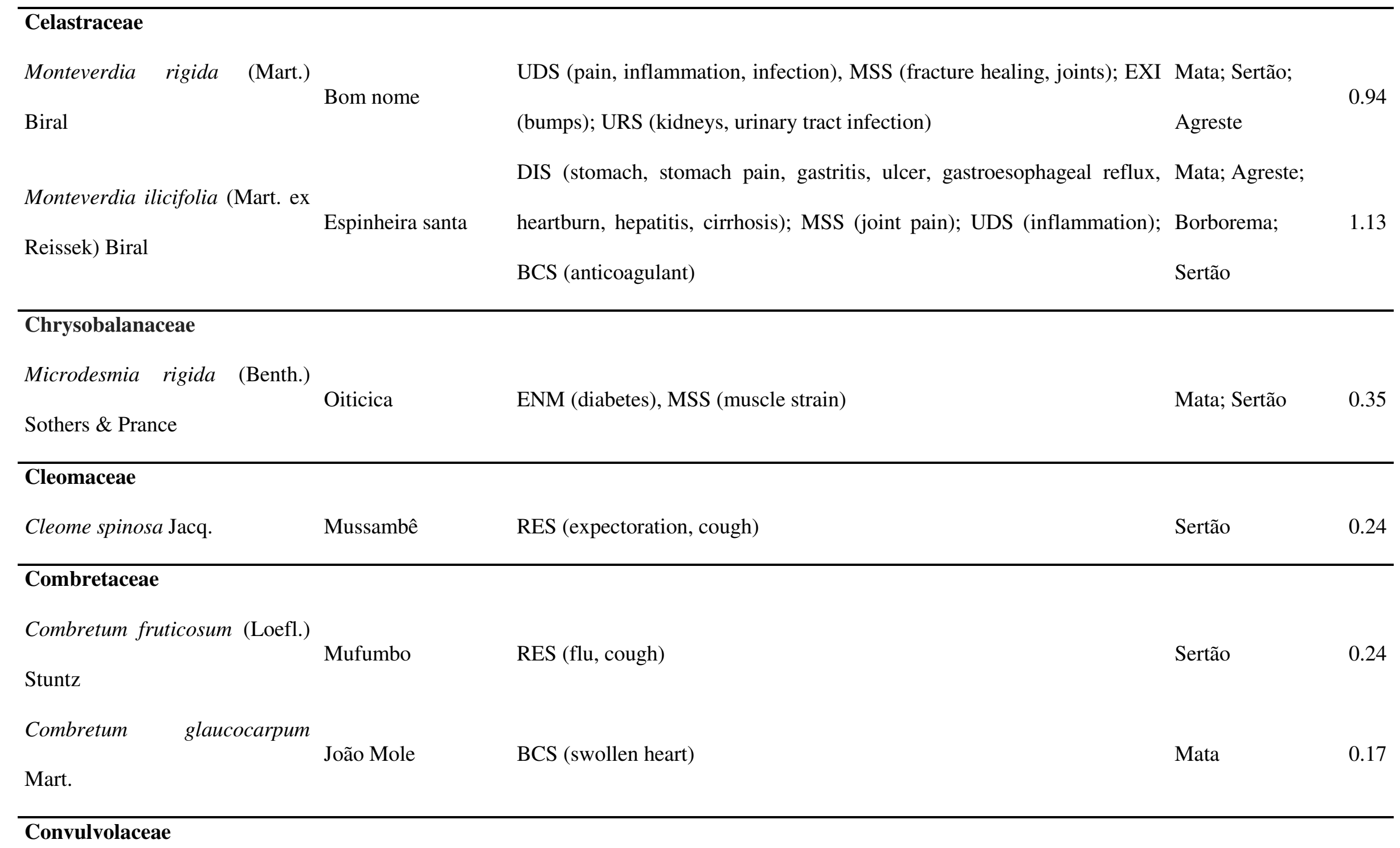


Operculina macrocarpa

(L.)

Batata de purga DIS (purgative), BCS (anticoagulant, hemorrhoids), UDS (inflammation)

Mata; Agreste;

Urb.

Sertão

Costaceae

Costus spicatus (Jacq.) Sw. Cana da índia URS (kidney problems, urinary tract infection)

Mata

0.24

\section{Cucurbitaceae}

Luffa operculata (L.) Cogn.

Cabacinha

RES (sinusitis); SST (shrink lumps)

Mata; Agreste;

Sertão

$\begin{array}{lll}\text { Momordica charantia L. } & \text { Melão de São } & \\ & \text { Caetano } & \text { BCS (hemorrhoids) } \\ & & \text { RSS (cysts, myoma), ENM (thyroid), URS (kidney stones), UDS }\end{array}$

Sicana odorifera (Vell.) Naudin Croá (inflammation)

Mata; Agreste

Mata; Agreste;

Wilbrandia sp.

Cabeça de negro

ENM (blood clotting), SST (shrink lumps)

Sertão

\section{Erythroxylaceae}

Erythroxylum sp.

Rompe gibão

MSS (back pain, joints), NES (nerves)

Sertão

0.41

\section{Equisetaceae}

Equisetum giganteum L. Cavalinha

URS (kidney stones, urinary tract infection, kidneys, diuretic), ENM Mata; Sertão 
(weight loss); UDS (infection); NEO (breast cysts); RSS (prostate); UDS

(inflammation)

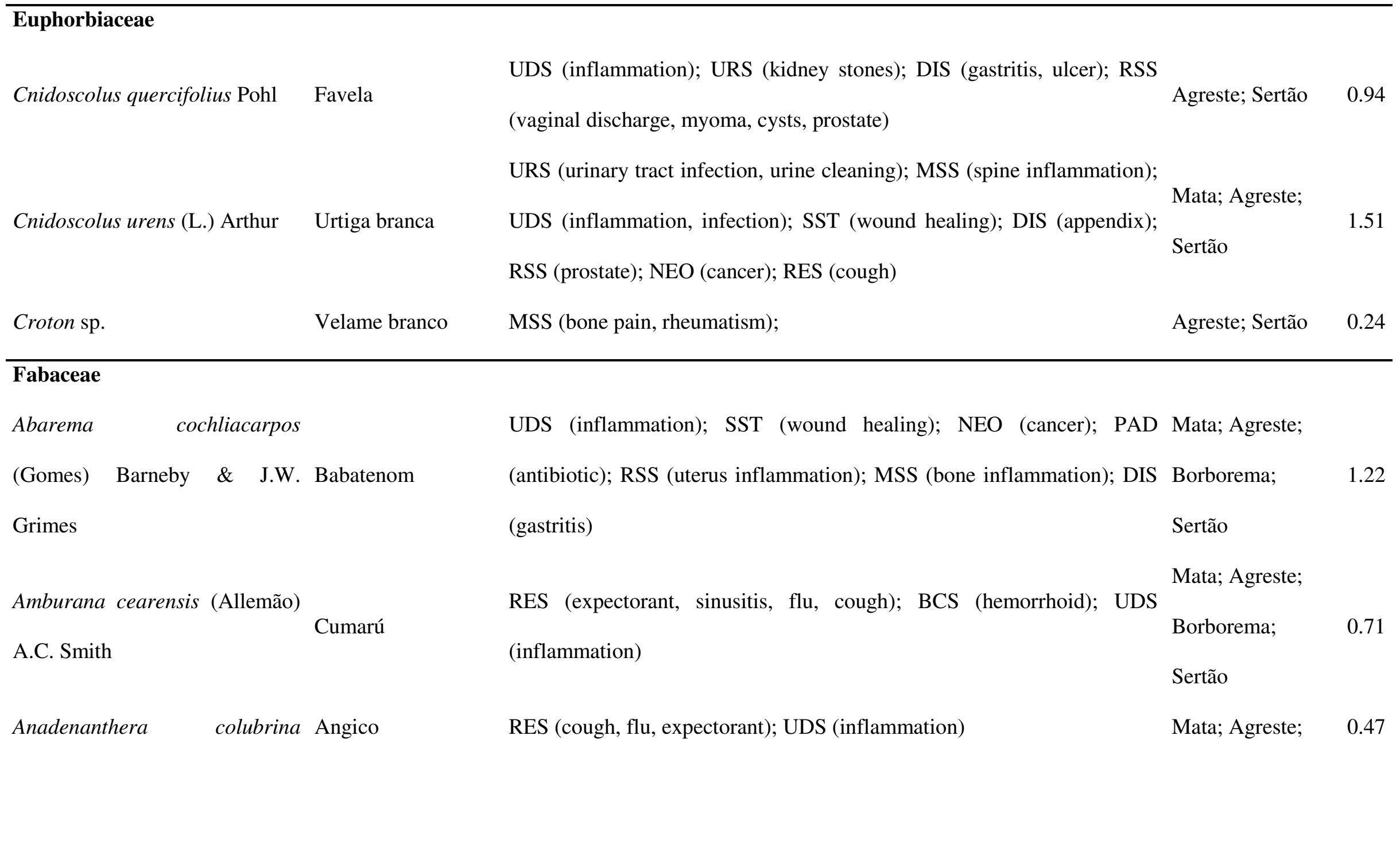


(Vell.) Brenan

Bauhinia sp. ${ }^{1}$

Bauhinia sp. ${ }^{2}$

Cajanus cajan (L.) Huth

Cenostigma pyramidale (Tul.)

E. Gagnon \& G.P. Lewis

Erythrina velutina Willd.

Glycyrrhiza glabra L.

Hymenaea courbaril L.

Libidibia ferrea (Mart. ex Tul.)

L.P. Queiroz
Mulungú

Jatobá

Mororó

Pata de vaca

Feijão gandú

Catingueira

Alcaçuz

Jucá
ENM (diabetes, cholesterol)

Mata; Sertão

Mata; Agreste;

ENM (diabetes, cholesterol); MSS (spine problems); UDS (inflammation)

NEO (intestinal cancer), ENM (diabetes), BCS (thrombosis)

Borborema;

Sertão

Mata

RES (cough, flu); DIS (bellyache); ENM (cholesterol)

Agreste; Sertão

Mata; Agreste;

NES (insomnia, tranquilizer, nerve weakness, memory); RES (cough)

Sertão

Mata

RES (expectoration)

RES (expectoration, cough, flu); RSS (prostate inflammation; cysts; Mata; Agreste;

erectile dysfunction); URS (kidney problems); BCS (anemia); ENM Borborema;

(fortifying, rickets) UDS (inflammation, pain)

Sertão

MSS (bone pain, spine, tendinitis, bursitis, spine inflammation); URS

(kidney pain); SST (lumps); RSS (cysts); UDS (inflammation); RES Mata; Agreste; Sertão (expectorant) 
Mimosa tenuiflora (Willd.) Poir. Jurema preta

$\begin{array}{ll}\text { Mimosa sp. } & \text { Malícia } \\ \text { Mucuna urens (L.) Medik. } & \text { Coronha } \\ \text { Myroxylon peruiferum L. f. } & \text { Bálsamo } \\ \text { Piptadenia sp. } & \text { Jurema bran } \\ & \\ \text { Ptredon emarginatus (Vogel.) } & \text { Sucupira } \\ \text { Kunth } & \end{array}$

Kunth

Senna sp.

Sena

Tamarindus indica $\mathrm{L}$

Tamarindo

BCS (anemia)

SST (wound healing)

UDS (inflammation, pain); MSS (herniated disc, spine inflammation)

RES (expectorant)

DIS (gastritis)

Mata; Sertão

Sertão

MSS (spine pain, spine inflammation, herniated disc, joint pain, bone pain, bone inflammation, osteoarthritis), DIS (sore throat); ENM (diabetes); BCS (thrombosis; high blood pressure); RES (sinusitis, tonsillitis); URS (kidneys)

Agreste; Sertão

Mata; Sertão

Sertão

DIS (indigestion; constipation, "release dry feces", intestinal colic); ENM Mata; Agreste; (weight loss); RES (cough); PAD (worm infection); UDS (fever, Borborema;

infection)

Sertão

Mata; Sertão

\section{Humiriaceae}

Endopleura uchi (Huber)

Cuatrec.

\section{Illiaceae}


Mata; Agreste;

Illicium verum Hooker

Anil estrelado

BCS (heart, high blood pressure); MSS (bone pain, back pain); DIS (indigestion, bellyache, liver, stomach ache); UDS (pain); RSS (colic)

Sertão

\begin{tabular}{|c|c|c|c|c|}
\hline \multicolumn{5}{|l|}{ Lamiaceae } \\
\hline Lavandula angustifolia Mill. & Alfazema & $\begin{array}{l}\text { DIS (baby colic, constipation, intestine); RES (cough); NES } \\
\text { (tranquilizer), UDS (colic, pain, fever), CUD ("evil eye"), BCS } \\
\text { (jaundice); PAD (infection) }\end{array}$ & $\begin{array}{l}\text { Mata; Agreste; } \\
\text { Borborema; } \\
\text { Sertão }\end{array}$ & 1.4 \\
\hline Melissa officinalis $\mathrm{L}$. & Melissa & NES (tranquilizer) & Mata & 0.17 \\
\hline Mentha sp. & Hortelã & $\begin{array}{l}\text { RES (flu, expectoration); ENM (weight loss); PAD ("bacteria in the } \\
\text { stomach"); BCS (prevent strokes) }\end{array}$ & Mata; Sertão & 0.76 \\
\hline $\begin{array}{l}\text { Mesosphaerum suaveolens (L.) } \\
\text { Kuntze }\end{array}$ & Alfazema braba & $\begin{array}{l}\text { DIS (bellyache, constipation, intestine); ENM (cholesterol, diabetes, } \\
\text { weight loss) }\end{array}$ & Sertão & 0.6 \\
\hline Ocimum sp. ${ }^{1}$ & Manjericão & CUD ("bad air"); RES (flu) & Mata; Sertão & 0.35 \\
\hline Ocimum sp. ${ }^{2}$ & Alfavaca & RES (sinusitis) & Mata & 0.17 \\
\hline Origanum sp. & Orégano & $\begin{array}{l}\text { ENM (menopause); NEO (cancer); DIS (constipation); NES (insomnia); } \\
\text { PAD (candidiasis); RES (cough) }\end{array}$ & $\begin{array}{l}\text { Mata; Agreste; } \\
\text { Sertão }\end{array}$ & 1.04 \\
\hline Rosmarinus officinalis $\mathrm{L}$. & Alecrim & BCS (arrhythmia, high blood pressure, heart problems, circulation, & Mata; Agreste; & 1.67 \\
\hline
\end{tabular}


CVA); DIS (stomach ache, constipation, indigestion); NES (depression, Borborema;

tranquilizer, meningitis); UDS (headache); RES (sinusitis, tiredness, Sertão

asthma); ENM (thyroid)

ENM (weight loss, appetite suppressant, menopause); DIS (intestinal

Salvia hispanica $\mathrm{L}$.

Chia

Sálvia

Salvia officinalis L.

Vitex gardneriana Schauer

Jaramataia

Lauraceae

Cinnamomum sp.

Canela

Laurus nobilis L.

Louro

Persea americana Mill.
Abacate
NES (tranquilizer, stimulant); ENM (weight loss, diabetes); BCS (low Mata; Agreste;

blood pressure, prevent blood clotting); DIS (throat, stomach ache); RES Borborema;

1.64

(hoarseness); UDS (vomiting); MSS (bones); EXI (bumps) Sertão

DIS (diarrhea, indigestion, stomach); UDS (headache); URS (kidneys); UDS (headache)

Mata; Agreste;

Borborema;

Sertão

Agreste;

Borborema;

BCS (heart); URS (kidneys) 
Sertão

\section{Lecythidaceae}

Bertholletia excelsa Bonpl

Castanha do Pará

ENM (liver fat)

Mata; Sertão

0.17

\section{Linaceae}

Linum usitatissimum $\mathrm{L}$.

Linhaça

DIS (constipation, intestinal regulation); BCS (thrombosis, CVA); MSS Mata; Agreste;

(rheumatism); ENM (weight loss)

Sertão

Lyrthaceae

DIS (sore throat, gastritis, stomach, heartburn); RES (hoarseness, cough; tonsillitis); RSS (prostate, erectile dysfunction); BCS (heart); ENM (liver

Punica granatum $\mathrm{L}$.

Romã

fat); UDS (inflammation); PAD (antibiotic); SST (wound healing); URS

Borborema;

(kidneys)

Sertão

\section{Malvaceae}

Abelmoschus esculentus (L.)

Moench

Quiabo

BCS (CVA); MSS (bones)

Sertão

Chorisia glaziovii (Kuntze) E.

Santos

Gossypium herbaceum L. 
ENM (diabetes, cholesterol, triglycerides, liver fat, weight loss); URS

Hibiscus sp

Hibisco

(diuretic); BCS (blood circulation, swelling, low immunity, blood

pressure, prevent blood clotting); UDS (inflammation); DIS (liver

inflammation)

Mata; Agreste;

Borborema;

Pseudobombax marginatum (A.

St.-Hil., Juss. \& Cambess.) A. Imbiratanha

Robyns

\section{Melastomataceae}

MSS (arthritis, osteoarthritis, bursitis, herniated disc, bone pain, joint Mata; Agreste;

Miconia albicans (Sw.) Triana Canela de velho pain, tendinitis, rheumatism, bone inflammation); UDS (pain, infection, Borborema;

headache, inflammation); PAD (Chikungunya)

Sertão

\section{Meliaceae}

Cedrela odorata $\mathrm{L}$.

Cedro

DIS (intestinal problems, constipation, bellyache); UDS (pain,

inflammation)

Mata; Sertão

\section{Menispermaceae}

Cissampelos sympodialis

Eichler 


\begin{tabular}{|c|c|c|c|c|}
\hline \multicolumn{5}{|l|}{ Monimiaceae } \\
\hline Peumus boldus Molina & Boldo do Chile & $\begin{array}{l}\text { DIS (diarrhea, liver problems, stomach ache, indigestion, intestine, } \\
\text { stomach problems, sulfur burps, gastritis); URS (kidneys); MBD } \\
\text { (hangover); ENM (liver fat) }\end{array}$ & $\begin{array}{l}\text { Mata; Agreste; } \\
\text { Borborema; } \\
\text { Sertão }\end{array}$ & 1.13 \\
\hline \multicolumn{5}{|l|}{ Moraceae } \\
\hline Brosimum gaudichaudii Trécul & Mamica de cadela & SST (Vitiligo) & Mata & 0.17 \\
\hline Morus sp. & Amora & $\begin{array}{l}\text { ENM (diabetes, menopause, weight loss, cholesterol); URS (diuretic); } \\
\text { BCS (boost immunity, high blood pressure); UDS (inflammation) }\end{array}$ & $\begin{array}{l}\text { Mata; Borborema; } \\
\text { Sertão }\end{array}$ & 0.94 \\
\hline \multicolumn{5}{|l|}{ Moringaceae } \\
\hline Moringa sp. & Moringa & NES (memory); NEO (cancer) & Sertão & 0.35 \\
\hline \multicolumn{5}{|l|}{ Musaceae } \\
\hline Musa $\mathrm{x}$ paradisiaca $\mathrm{L}$. & Banana & RES (cough) & Sertão & 0.17 \\
\hline \multicolumn{5}{|l|}{ Myristicaceae } \\
\hline Myristica fragans Houtt & Noz moscada & SES (labyrinthitis); BCS (prevent strokes) & $\begin{array}{l}\text { Mata; Agreste; } \\
\text { Sertão }\end{array}$ & 0.35 \\
\hline
\end{tabular}

\section{Myrtaceae}

Eucalyptus globulus Labill. Eucalipto

RES (sinusitis, common cold, flu, expectorant); NES (tranquilizer); DIS Mata; Agreste; 
(bellyache); UDS (fever); MSS (bone pain)

Eugenia uniflora L.

Myrcia speciosa

McVaugh

Psidium guajava L.

Guava

Syzygium aromaticum

(L.)

Merr. \& L. M. Perry

Syzygium cumini (L.) Skeels

Oliveira

DIS (bellyache)

Pedra-hume-kar ENM (diabetes)

DIS (bellyache)

pressure)

ENM (weight loss, "reduce the levels"); NEO (cancer)

Sertão

Mata

Sertão

UDS (headache, dizziness, bad breath); DIS (indigestion; toothache); Mata; Agreste;

SES (Labyrinthitis); NES (Insomnia, tranquilizer); BCS (high blood Borborema;

Sertão

Sertão

\section{Nyctaginaceae}

Boerhavia diffusa $\mathrm{L}$.

Pega pinto

URS (kidneys, urinary tract infection)

Sertão

Olacaceae

Ximenia americana

Ximenia americana L. Ameixa

DIS (gastritis); UDS (inflammation); SST (wound healing); ENM (cholesterol)

Mata; Agreste;

Borborema;

0.69

Sertão

\section{Opiliaceae}


Agonandra brasiliensis Miers

ex Benth. \& Hook. f.

\begin{tabular}{|c|c|c|c|c|}
\hline \multicolumn{5}{|l|}{ Papaveraceea } \\
\hline Argemone mexicana $\mathrm{L}$. & Cardo santo & BCS (CVA, thrombosis) & $\begin{array}{l}\text { Mata; Agreste; } \\
\text { and Sertão }\end{array}$ & 0.24 \\
\hline \multicolumn{5}{|l|}{ Passifloraceae } \\
\hline Turnera subulata Smith. & Chanana & URS (urinary tract infection) & Sertão & 0.17 \\
\hline \multicolumn{5}{|l|}{ Pedaliaceae } \\
\hline Sesamum orientale $\mathrm{L}$. & Gergelim preto & $\begin{array}{l}\text { BCS (thrombosis, CVA); MSS (bone pain, joint pain, rheumatism, bone } \\
\text { calcium); UDS (numbness); DIS (intestinal regulation); ENM } \\
\text { (menopause, appetite suppressant, weight loss) }\end{array}$ & $\begin{array}{l}\text { Mata; Agreste; } \\
\text { Sertão }\end{array}$ & 1.24 \\
\hline \multicolumn{5}{|l|}{ Petiveriaceae } \\
\hline Petiveria alliacea $\mathrm{L}$. & Tipi & MSS (rheumatism) & Mata; Sertão & 0.17 \\
\hline \multicolumn{5}{|l|}{ Phyllanthaceae } \\
\hline Phyllanthus niruri L. & Quebra pedra & $\begin{array}{l}\text { URS (kidney stones, kidney problems); DIS (liver, gallstones); EXI } \\
\text { (bumps) }\end{array}$ & $\begin{array}{l}\text { Mata; Borborema; } \\
\text { Sertão }\end{array}$ & 0.65 \\
\hline
\end{tabular}

\section{Piperaceae}


Sertão

\begin{tabular}{|c|c|c|c|c|}
\hline Poaceae & & & & \\
\hline $\begin{array}{l}\text { Cymbopogon citratus (DC.) } \\
\text { Stapf }\end{array}$ & Capim santo & $\begin{array}{l}\text { NES (tranquilizer, stimulant); DIS (bellyache); BCS (high blood } \\
\text { pressure) }\end{array}$ & $\begin{array}{l}\text { Mata; Agreste; } \\
\text { Sertão }\end{array}$ & 0.58 \\
\hline Zea mays L. & Milho & URS (kidneys), BCS (jaundice) & Sertão & 0.35 \\
\hline Polygonaceae & & & & \\
\hline $\begin{array}{l}\text { Polygonum } \\
\text { Michx. }\end{array}$ & Erva de bicho & URS (kidneys) & Mata & 0.17 \\
\hline Rhamnaceae & & & & \\
\hline Ziziphus joazeiro Mart. & Juá & SST (dandruff); DIS (gum disease) & Mata; Sertão & 0.35 \\
\hline Rubiaceae & & & & \\
\hline $\begin{array}{l}\text { Coutarea hexandra (Jacq.) K. } \\
\text { Schum. }\end{array}$ & Quina quina & $\begin{array}{l}\text { ENM (diabetes, blood clotting), RES (sinusitis), MSS (rheumatism), } \\
\text { UDS (fever) }\end{array}$ & $\begin{array}{l}\text { Mata; Agreste; } \\
\text { Sertão }\end{array}$ & 0.76 \\
\hline Genipa americana $\mathrm{L}$. & Jenipapo & MSS (fracture healing); BCS (increase blood platelets) & Sertão & 0.35 \\
\hline Guettarda sp. & Angélica & RSS (menstrual cramps) & Sertão & 0.17 \\
\hline Morinda citrifolia $\mathrm{L}$. & Noni & ENM (diabetes, weight loss); NEO (cancer); BCS (blood circulation; & Agreste; Sertão & 1.12 \\
\hline
\end{tabular}


fluid retention, hemorrhoids, high blood pressure); DIS (gastritis); RSS

(uterus inflammation)

UDS (inflammation); RSS (cysts, nodule, myoma, polycysts, uterus Mata; Agreste;

Uncaria sp.

Unha de gato

inflammation); MSS (bone inflammation)

\section{Rutaceae}

Citrus aurantium L.

Laranja

NES (tranquilizer)

Mata; Sertão

0.17

Pilocarpus sp.

Jaborandi

UDS (fever)

Mata

\section{Sapotaceae}

Sideroxylon obtusifolium (Roem

\& Schult.) T.D. Penn.

Quixaba

UDS (inflammation, pain); MSS (spine inflammation); SST (wound

Mata; Agreste;

Borborema; healing); URS (kidneys); EXI (bumps)

Sertão

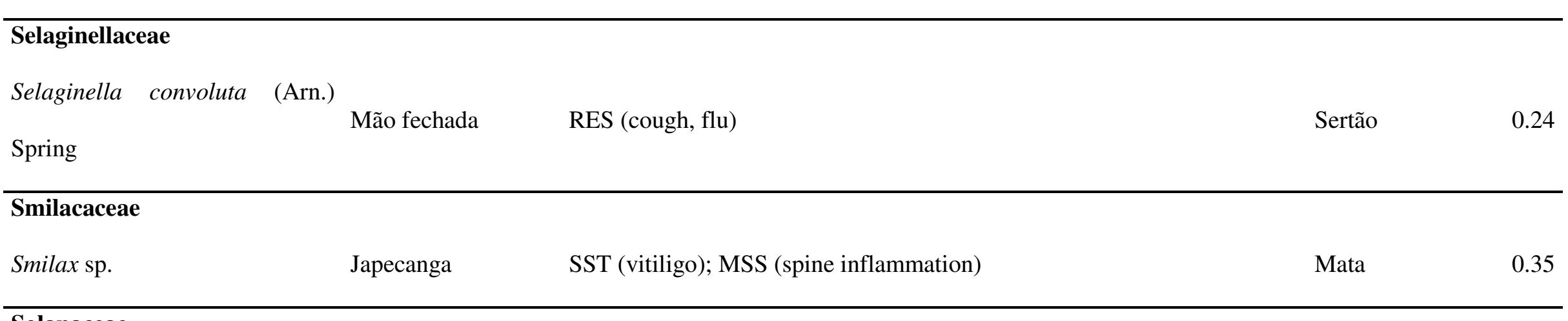

Solanaceae 
Theaceae

Chá

preto

ENM (cholesterol, diuretic, weight loss, loss of appetite); NES Mata; Agreste; verde/Chá

Sertão

\section{Verbenaceae}

Lippia alba (Mill.) N.E. Br. ex

Britton \& P. Wilson

Erva cidreira

RES (expectoration); BCS (anemia); DIS (indigestion, bellyache); NES Mata; Agreste;

(tranquilizer, insomnia); ENM (whet the appetite)

Sertão

\section{Violaceae}

Pombalia lanata (A. St.-Hil.)

Paula-Souza

Papaconha

RES (expectoration, cough, flu); PAD (worm infection); UDS (fever)

Mata; Borborema;

Sertão

\section{Vitaceae}

Cissus sp.

Parreira

URS (kidneys); MSS (spine)

Sertão

0.35

Zingiberaceae

Alpinia zerumbet (Pers.) B.L.

Burtt \& R.M. Sm.

UDS (fever); RES (expectoration) 
Curcuma longa $\mathrm{L}$.

Cúrcuma

Zingiber officinale Roscoe

Gengibre
UDS (inflammation); BCS (hepatitis; jaundice); PAD (antibiotic); RES (flu); MSS (bones)

BCS (high blood pressure, prevent blood clotting); DIS (throat pain, sore throat, stomach); RES (cough, expectoration, flu, hoarseness); ENM (liver fat, cholesterol, weight loss); UDS (pain, inflammation); NES Borborema; (stimulant); URS (diuretic)

Sertão

\begin{tabular}{|c|c|c|c|c|}
\hline \multicolumn{5}{|l|}{ Indeterminated } \\
\hline Indeterminated 1 & Baço & RES (cough, flu, tiredness), UDS (pain) & $\begin{array}{l}\text { Borborema; } \\
\text { Sertão }\end{array}$ & 0.47 \\
\hline Indeterminated 2 & Caninana & SST (wound healing) & Sertão & 0.17 \\
\hline Indeterminated 3 & Catinga branca & DIS (diarrhea) & Borborema & 0.17 \\
\hline Indeterminated 4 & Cauaçú & DIS (gastritis, ulcer) & Borborema & 0.24 \\
\hline Indeterminated 5 & $\begin{array}{l}\text { Chocalho } \\
\text { vaqueiro }\end{array}$ & MSS (bone pain, joint pain, rheumatism) & Sertão & 0.3 \\
\hline Indeterminated 6 & Cipó de cruz & MSS (joints, rheumatism, spine), UDS (inflammation) & Sertão & 0.47 \\
\hline Indeterminated 7 & Espriteira & RES (common cold, flu) & Agreste & 0.24 \\
\hline Indeterminated 8 & Jalapa & ENM (diabetes) & Mata & \\
\hline
\end{tabular}




\begin{tabular}{|c|c|c|c|c|}
\hline Indeterminated 9 & Junço & UDS pain) & Sertão & 0.17 \\
\hline Indeterminated 10 & Mapirunga & MSS (rheumatism), UDS (pain) & Agreste & 0.28 \\
\hline Indeterminated 11 & Maria leite & URS (kidney stones), DIS (gallstone) & Mata & 0.34 \\
\hline Indeterminated 12 & Pau tenente & ENM (diabetes, cholesterol), UDS (inflammation) & Mata; Sertão & 0.41 \\
\hline Indeterminated 13 & Pimenta parda & RES (throat) & Agreste & 0.17 \\
\hline Indeterminated 14 & Porangaba & ENM (cholesterol, blood clotting, diabetes), DIS (intestine) & $\begin{array}{l}\text { Mata; Agreste; } \\
\text { Sertão }\end{array}$ & 0.47 \\
\hline Indeterminated 15 & Quebra faca & ENM (diabetes, cholesterol), SST (pruritus) & Sertão & 0.41 \\
\hline Indeterminated 16 & Sassafrás & URS (urinary tract infection, kidneys) & Sertão & 0.24 \\
\hline Indeterminated 17 & Urinana & URS (urinary tract infection), ENM (retained fat) & Mata & 0.35 \\
\hline
\end{tabular}


Table 2. Medicinal plants of high Relative Importance in public markets of different Mesoregions in the state of Paraíba, Northeastern Brazil. $\mathrm{N}=$ species native to Brazil. $\mathrm{E}=$ exotic species. $\mathrm{RI}=$ Relative Importance.

\begin{tabular}{|c|c|c|c|c|c|}
\hline Species & Origin & RI & Species & Origin & RI \\
\hline Punica granatum & $\mathrm{E}$ & 2 & Abarema cochliacarpos & $\mathrm{N}$ & 1.22 \\
\hline Zingiber officinale & $\mathrm{E}$ & 1.78 & Miconia albicans & $\mathrm{N}$ & 1.21 \\
\hline Myracrodruon urundeuva & $\mathrm{N}$ & 1.69 & Illicium verum & $\mathrm{E}$ & 1.18 \\
\hline Rosmarinus officinalis & $\mathrm{E}$ & 1.67 & Syzygium aromaticum & $\mathrm{E}$ & 1.18 \\
\hline Cinnamomum sp. & $\mathrm{E}$ & 1.64 & Anacardium occidentale & $\mathrm{N}$ & 1.17 \\
\hline Foeniculum vulgare & $\mathrm{E}$ & 1.59 & Matricaria chamomilla & $\mathrm{E}$ & 1.17 \\
\hline Pimpinella anisum & $\mathrm{E}$ & 1.59 & Equisetum giganteum & $\mathrm{N}$ & 1.17 \\
\hline Ptredon emarginatus & $\mathrm{N}$ & 1.54 & Monteverdia ilicifolia & $\mathrm{N}$ & 1.13 \\
\hline Cnidoscolus urens & $\mathrm{N}$ & 1.51 & Peumus boldus & $\mathrm{E}$ & 1.13 \\
\hline Hymenaea courbaril & $\mathrm{N}$ & 1.42 & Senna sp. & $\mathrm{N}$ & 1.12 \\
\hline Camellia sinensis & $\mathrm{E}$ & 1.42 & Morinda citrifolia & $\mathrm{E}$ & 1.12 \\
\hline Lavandula angustifolia & $\mathrm{E}$ & 1.4 & Allium sp. & $\mathrm{E}$ & 1.07 \\
\hline Anethum graveolens & $\mathrm{E}$ & 1.37 & Allium sativum & $\mathrm{E}$ & 1.06 \\
\hline Hibiscus sp. & $\mathrm{E}$ & 1.37 & Coriandrum sativum & $\mathrm{E}$ & 1.06 \\
\hline Libidibia ferrea & $\mathrm{N}$ & 1.29 & Eucalyptus globulus & $\mathrm{E}$ & 1.06 \\
\hline Sesamum orientale & $\mathrm{E}$ & 1.24 & Origanum sp. & $\mathrm{E}$ & 1.04 \\
\hline
\end{tabular}

During the study period 88 species were unavailable at least once at least at one of the traders interveiwed (Table 3). The Mata was the mesoregion where the highest number of species was absent at some point during the year, while the Borborema was the region with least seasonal absence of species. 
Table 3. Percentage of informants that reported the absence of some species during the interview period for each studied mesoregion. Blank cells indicate that the species was not recorded in the mesoregion at any time of the year.

\begin{tabular}{|c|c|c|c|c|c|c|c|c|c|c|c|c|c|c|c|c|}
\hline \multirow[t]{2}{*}{ Species } & \multicolumn{4}{|c|}{$\begin{array}{c}\text { Mata } \\
(\mathrm{N}=13)\end{array}$} & \multicolumn{4}{|c|}{$\begin{array}{l}\text { Agreste } \\
(\mathrm{N}=10)\end{array}$} & \multicolumn{4}{|c|}{$\begin{array}{c}\text { Borborema } \\
\qquad(N=4)\end{array}$} & \multicolumn{4}{|c|}{$\begin{array}{l}\text { Sertão } \\
(\mathrm{N}=8)\end{array}$} \\
\hline & $1^{\circ}$ & $2^{\circ}$ & $3^{\circ}$ & $4^{\circ}$ & $1^{\circ}$ & $\mathbf{2}^{\circ}$ & $\mathbf{3}^{\circ}$ & & $1^{\circ}$ & $\mathbf{2}^{\circ}$ & $\mathbf{3}^{\circ}$ & $4^{\circ}$ & $1^{\circ}$ & $2^{\circ}$ & $\mathbf{3}^{\circ}$ & $4^{\circ}$ \\
\hline Abarema cochliacarpos & 7.69 & - & - & - & 30 & 20 & 20 & 10 & - & - & - & - & - & 12.5 & - & - \\
\hline Agave sp. & & & & & - & - & - & 10 & & & & & - & - & - & - \\
\hline Ageratum conyzoides & & & & & & & & & & & & & - & 12.5 & 12.5 & 12.5 \\
\hline Allium sp. & 7.69 & - & 15.38 & 7.69 & - & 30 & 10 & 10 & - & - & 25 & - & - & 25 & 12.5 & 25 \\
\hline Aloe vera & 7.69 & - & 15.38 & 7.69 & & & & & 25 & - & 25 & - & & & & \\
\hline Alpinia zerumbet & 15.38 & 7.69 & - & - & & & & & & & & & - & 12.5 & - & - \\
\hline Amburana cearensis & 15.38 & - & 7.69 & 15.38 & 10 & - & 30 & - & - & - & - & - & - & - & - & - \\
\hline Anacardium occidentale & - & - & 7.69 & - & - & - & 10 & 10 & - & - & - & - & - & 12.5 & 12.5 & - \\
\hline $\begin{array}{l}\text { Anadenanthera } \\
\text { colubrina }\end{array}$ & 15.38 & - & - & 7.69 & - & 10 & - & - & & & & & - & - & - & - \\
\hline Anemopaegma sp. & 7.69 & 7.69 & - & - & & & & & & & & & - & - & 12.5 & - \\
\hline Anethum graveolens & 23.07 & - & - & - & - & - & 20 & 20 & - & - & - & - & - & 12.5 & 12.5 & - \\
\hline$\overline{\text { Annona muricata }}$ & 7.69 & - & 15.38 & - & - & - & - & - & - & - & - & - & - & - & - & - \\
\hline Baccharis sp. & 15.38 & 7.69 & 15.38 & - & - & - & - & - & - & - & 50 & - & 25 & - & 12.5 & - \\
\hline$\overline{\text { Bahuinia sp. }}{ }^{2}$ & 7.69 & 7.69 & - & - & 10 & - & 10 & - & - & - & - & - & - & - & - & - \\
\hline Bertholletia excelsa & - & - & 7.69 & - & & & & & & & & & - & - & - & - \\
\hline Boerhavia coccinea & - & - & 7.69 & - & & & & & & & & & - & - & 12.5 & 12.5 \\
\hline$\overline{\text { Brassica } \mathrm{sp} .}$ & - & - & - & - & 10 & 10 & - & - & - & - & - & - & - & - & 12.5 & 12.5 \\
\hline Camellia sinensis & 7.69 & - & - & - & 10 & 20 & - & - & - & - & 25 & 25 & 25 & 12.5 & - & 12.5 \\
\hline Caryocar sp. & & & & & & & & & & & & & - & - & 12.5 & 12.5 \\
\hline
\end{tabular}




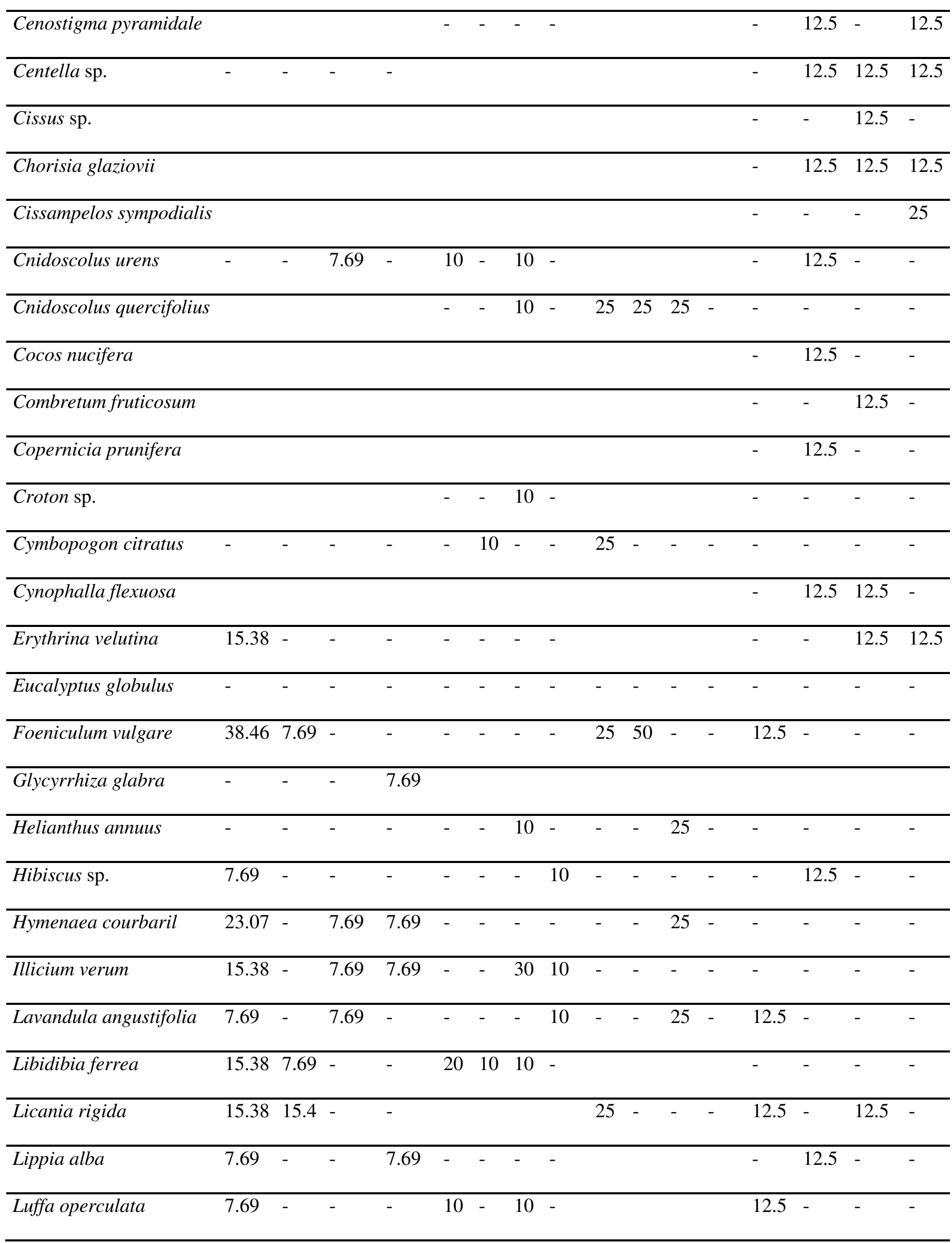




\begin{tabular}{|c|c|c|c|c|c|c|c|c|c|c|c|c|c|c|c|c|}
\hline Maytenus rigida & - & - & - & - & - & - & - & - & & & & & - & - & 12.5 & - \\
\hline Mentha sp. & - & - & - & - & & & & & 25 & 25 & 25 & - & - & - & - & - \\
\hline Miconia albicans & - & - & 7.69 & 7.69 & - & - & 10 & - & - & - & - & - & - & - & - & - \\
\hline Monteverdia ilicifolia & - & - & - & 7.69 & - & 10 & - & - & - & - & - & - & - & - & - & 12.5 \\
\hline Morus sp. & 7.69 & - & - & 7.69 & & & & & - & - & - & - & - & - & - & - \\
\hline Musa $\mathrm{x}$ paradisíaca & & & & & & & & & & & & & - & 12.5 & - & 12.5 \\
\hline Myracrodruon & & & & & & & & & & & & & & & & \\
\hline urundeuva & - & - & - & - & 30 & 10 & 20 & - & & & & & - & - & - & - \\
\hline Persea americana & & & & & - & - & - & - & - & - & - & - & - & - & - & 12.5 \\
\hline Phyllanthus niruri & 23.07 & 15.4 & - & 30.77 & 10 & - & - & 10 & - & - & 25 & - & - & - & - & - \\
\hline Pimpinella anisum & - & - & - & 7.69 & - & - & - & - & - & - & - & - & - & - & - & - \\
\hline$\overline{P o m b a l i a ~ l a n a t a}$ & - & - & 23.08 & - & & & 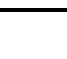 & & - & - & - & - & - & 12.5 & 25 & 12.5 \\
\hline Ptredon emarginatus & 7.69 & 7.69 & 7.69 & - & 10 & 10 & 20 & 10 & 25 & - & - & - & - & - & - & - \\
\hline Punica granatum & 7.69 & - & - & - & 10 & - & - & - & - & - & - & - & - & - & - & 25 \\
\hline Rosmarinus officinalis & - & - & 7.69 & - & 10 & - & - & - & - & - & - & - & 12.5 & 12.5 & 12.5 & - \\
\hline Salvia hispânica & 7.69 & - & - & - & - & 10 & - & - & - & - & 25 & - & - & - & 12.5 & - \\
\hline Sambucus australis & 7.69 & - & - & - & 10 & - & 10 & 10 & - & - & 25 & 25 & - & - & - & - \\
\hline$\overline{\text { Schnopsis brasiliensis }}$ & - & - & - & - & - & 10 & 10 & 10 & & & & & - & - & 12.5 & 12.5 \\
\hline Senna sp. & 7.69 & - & 7.69 & - & 10 & - & 10 & - & - & - & - & - & - & - & - & - \\
\hline Sesamum orientale & - & - & - & - & 10 & - & - & - & 25 & 25 & 25 & - & - & - & 12.5 & 25 \\
\hline Sideroxylon obtusifolium & - & - & - & - & - & 10 & 10 & 10 & - & - & 25 & - & - & - & - & - \\
\hline Solanum americanum & & & & & & & & & & & & & - & 12.5 & - & - \\
\hline Symphytum officinale & - & - & - & - & & & & & & & & & - & - & 12.5 & 12.5 \\
\hline$\overline{\text { Syzygium cumini }}$ & - & - & - & - & - & - & - & - & - & - & - & - & - & 12.5 & - & - \\
\hline Tamarindus indica & 7.69 & 7.69 & - & - & & & & & & & & & - & 12.5 & 12.5 & 12.5 \\
\hline Taraxacum officinale & - & - & - & - & & & & & - & - & 25 & - & 25 & 25 & 12.5 & 12.5 \\
\hline
\end{tabular}




\begin{tabular}{|c|c|c|c|c|c|c|c|c|c|c|c|c|c|c|c|c|}
\hline Valeriana sp. & - & 7.69 & - & - & & & & & & & & & & & & \\
\hline Wilbrandia sp. & - & - & - & 7.69 & - & - & - & - & & & & & - & - & - & - \\
\hline Ximenia americana & - & - & - & - & - & - & - & - & - & - & 25 & - & - & - & - & - \\
\hline Zingiber officinale & - & - & 7.69 & - & - & - & - & - & - & - & - & - & - & - & 12.5 & - \\
\hline Ziziphus joazeiro & - & - & - & 7.69 & & & & & & & & & - & 12.5 & - & - \\
\hline
\end{tabular}

\section{Discussion}

The medicinal plant species traded in the public markets of Paraíba were shared among the mesoregions, except for the Sertão, which had a more specific group of plants for sale. Climatic and seasonal variations did not seem to greatly influence plant availability throughout the year, considering that traders in general keep a stock of dry plants to ensure the supply of most species. Overall, leaves, bark, and seeds were the most frequently raded plant parts, with the bark predominating in the Agreste mesoregion, while leaves were the most traded plant structure in the remaining mesoregions.

The highest Relative Importance values were recorded for Punica granatum, Zingiber officinale, and Myracrodruon urundeuva. Although these species were recorded in all studied mesoregions and kept a relatively frequent availability in the trading locations during the research, previous studies involving some of the studied municipalities did not record these species in the market. In Guarabira, $P$. granatum was not previously recorded among the main species, and Z. officinale and M. urundeuva were also not recorded in a previous study conducted in the market of Patos [24], even though M. urundeuva occurs naturally in the region $[42,43]$. These data may indicate that over the past decade changes have occurred in plant availability or in the local importance of medicinal plants traded in these markets. It is worth mentiuoning that these species have ben commonly documented in markets of nearby regions, such as in Pernambuco, although $P$. granatum and Z. officinale usually presented relatively low RI values compared to what was observed in our study $[10,18,23]$. 
In most cases the medicinal use of leaves, bark, and seeds (Figure 3) was recorded, similarly to several other studies $[7,30,44]$. Previously a greater use of leaves and herbaceous plants in wetter regions, such as the Atlantic Forest has been commonly registered $[4,45,46]$, while in drier regions, such as the Caatinga, a predominance of the use of barks and woody plants has been shown [3,47], highlighting a relationship with the loss of foliage in the vegetation during the drier periods [16]. This apparent correspondence between the most used plant parts and the environment can also be seen in other studies conducted in dry $[6,8,48]$ and wetter environments $[33,20]$. However, in this study, it was not possible to establish a similar relationship between the environment and the most traded plant parts, and the Agreste was the only drier region where the bark was the main plant structure traded. The leaves were the main plant part in the remaining mesoregions, even in drier areas, where a more significant bark trade would have been expected. A possible explanation for that is the dynamism of medicinal plant trade, which involves not only the local plant species in the studied markets but also species from other regions and even imported from other countries, since, as observed here, most species with high Relative Importance were exotic.

It was impossible to establish a relationship between the unavailability of any species during some period and the mesoregion, given that when one species was unavailable in one trade location (market stand), it could usually be found in other locations of the same market. While there might be a relationship between plant habit and availability, given most species that showed some period of unavailability are herbaceous, similar to other studies [10], most species are sold dried, giving the traders the possibility to simply acquire and stock the material avoid a lack of the product [20], although at times traders simply might not have the financial resources to stock material.

In some cases, the informants stated that Foeniculum vulgare, according to them, cultivated in the Brejo and Curimataú areas (Agreste Mesoregion), was not available during 
some periods. A possible explanation for species unavailability in some cases could be traced to the recent sale of the whole stock by a trader, or the lack of interest by the trader in stocking a particular product given the low demand.

It was also possible to note the incorporation of a new species into the plant trade during our study: In the first stage of the interviews, Miconia albicans was only found in a few places of the Mata and Sertão mesoregions, and then a fast spread of this species in the market was observed. Although being a native species and, according to some informants, common in woody areas of both the Atlantic Forest and the Caatinga, its medicinal use was not well known until recently. According to the traders, the recent increase in the trade of this species occurred due to its recent promotion treat pain and muscular and rheumatic diseases on the internet and television, which led increasing consumer demand. Previous ethnobotanical studies did record this species, but made no reference to its medicinal use [49-51]. Previous records of its medicinal exist from Mexico [52], and five different species of Miconia have been reported as medicinal plants in Bolivia [5]. Similar cases have also ben reorted for Hibiscus sp., Camellia sinensis, and Zingiber officinale, species that began to be traded less than a decade ago, according to the informants, also influenced by the media and the internet.

The greater use of medicinal plants to treat diseases of the digestive and respiratory systems has often been reported in ethnobotanical studies [3,14,15,21], and explained by the fact that these diseases are most commonly affecting the population $[3,15]$. The emphasis on endocrine, nutritional, and metabolic diseases might partly be explained due to plant use for weight loss (Table 1), which, according to the traders, is also a consequence of the growing interest of customers in using plants that aid in losing weight and keeping a good shape. This has been related to the current habits of society, which tends to be sedentary and ingest highly caloric foods, becoming obese, and social media and television promoting the sale of medicinal plants for losing weight [53-56]. 
The four mesoregions shared 35 species. The one-way ANOSIM multivariate analysis demonstrated significant similarity between the mesoregions, except for the Sertão, which showed a significant differences from all remaining mesoregions.

The difference between the Sertão and the remaining mesoregions could also be observed in the Principal Coordinates Analysis. Interestingly, the Agreste Mesoregion, geographically located in a transition area between the Atlantic Forest and the Caatinga, fell in the center of the graph, sharing its limits with all remaining mesoregions. This leads us to suggest that the Agreste mesoregion represents a type of "biocultural ecotone" or "cultotone," resembling the concept of ecotone, understood not only as a transition area for vegetation, but of knowledge and practice in the use of medicinal plants in relation to the remaining mesoregions. Considering the relatively small territory of the state of Paraíba $\left(56,585 \mathrm{~km}^{2}\right)$ [35], it may be inferred that there is a permutation of knowledge and traded species between the different regions. Although a study with similarity analysis between different phytophysiognomies in the states of Paraíba and Pernambuco has not found similarity between the studied phytophysiognomies [57], it is worth noting that, in addition to the possibility of permutation of native species of different phytophysiognomies, the use of exotic species is a factor that collaborates for a greater sharing of species, even in so different areas as the Atlantic Forest and the Caatinga.

\section{Conclusions}

The inventory of medicinal plants available in the markets of Paraíba varies little throughout the year. In general, traders seem to keep permanent stocks of the main plants. Traders were also receptive to incorporatning new plants into their stocks, which might be explained several factors, such as the influence of the media and the internet, fostering the growing interest of customers in ceryain species. 
It was impossible to establish a relationship between the periods of species absence in some trading locations and the mesoregion where this absence occurred. The absence during certain periods is probably more related to temporary unavailability or the impossibility for the trader to stock the product, or moght even be related to environmental changes, which may influence species availability.

The Sertão mesoregion was the only one that showed a significant variation in teh inventory of species sold by the traders. It is also interesting that the Agreste mesoregion, geographically located in an intermediate region between the Atlantic Forest and the Caatinga, showed an intermediate similarity pattern with the remaining mesoregions.

\section{Declarations}

\section{Ethics approval and consent to participate}

The aim of this study was explained to each informant, who was then asked to sign a consent form, as required by the National Health Council and the Research Ethics Committee (Resolution 466/12). This research was approved by the Research Ethics Committee of the State University of Paraíba (Protocol No. 82943618.0.0000.5188). The authors interviewed 35 informants, who were informed of the aim of this scientific research and agreed to sign an informed consent form, according to necessary standards, and as described in the methodology through the protocol number.

\section{Consent for publication}

The participants who signed the consent form were aware that this scientific research could be published in the academic milieu.

\section{Availability of data and materials}

The datasets used and/or analysed during the current study are available from the 
corresponding author on reasonable request.

\section{Competing interests}

The authors declare that they have no competing interests.

\section{Funding}

Data of the present study are derived from the first author's master's thesis, which received a scholarship from the Conselho Nacional de Desenvolvimento Científico e Tecnológico $(\mathrm{CNPq})$.

\section{Authors' contributions}

ECF collected and analyzed the data and wrote the manuscript, DDC and RFPL coordinated the field research and the writing of the manuscript, RWB and NYPZ contributed in final write up.

\section{Acknowledgements}

The authors would like to thank all local traders for their reception and for agreeing to participate in the study.

\section{References}

1. Ribeiro RV, Bieski IGC, Balogun SO, Martins DT de O. Ethnobotanical study of medicinal plants used by Ribeirinhos in the North Araguaia microregion, Mato Grosso, Brazil. J Ethnopharmacol. 2017;205:69-102.

2. Silva TC, Silva JM, Ramos MA. What factors guide the selection of medicinal plants in a local pharmacopoeia? A case study in a rural community from a historically transformed atlantic forest landscape. Evidence-based Complement Altern Med. Hindawi; 2018;2018.

3. Coutinho PC, Soares ZA, Ferreira EC, Souza DV, Oliveira RS, Lucena RFP. Knowledge 
and use of medicinal plants in the Semiarid Region of Brazil. Brazilian J Biol Sci. 2015;2:5174.

4. Beltreschi L, Lima RB, Cruz DD. Traditional botanical knowledge of medicinal plants in a " quilombola " community in the Atlantic Forest of northeastern Brazil. Environ Dev Sustain. 2018. https://doi.org/10.1007/s10668-017-0079-6

5. Thomas E, Semo L, Morales M, Noza Z, Nuñez H, Cayuba A, et al. Ethnomedicinal practices and medicinal plant knowledge of the Yuracarés and Trinitarios from Indigenous Territory and National Park Isiboro-Sécure, Bolivian Amazon. J Ethnopharmacol. 2011;133:153-63.

6. Miara MD, Teixidor-Toneu I, Sahnoun T, Bendif H, Ait Hammou M. Herbal remedies and traditional knowledge of the Tuareg community in the region of Illizi (Algerian Sahara). J Arid Environ. 2019;167:65-73.

7. Amri E, Kisangau DP. Ethnomedicinal study of plants used in villages around Kimboza forest reserve in Morogoro, Tanzania. J Ethnobiol Ethnomed. 2012;8:1.

8. Tounekti T, Mahdhi M, Khemira H. Ethnobotanical study of indigenous medicinal plants of Jazan region, Saudi Arabia. Evidence-based Complement Altern Med. 2019;2019.

9. Santos ABN, Araújo MP, Sousa RS, Lemos JR, Santos ABN, Araújo MP, et al. Plantas medicinais conhecidas na zona urbana de Cajueiro da Praia, Piauí, Nordeste do Brasil. Rev Bras Plantas Med. 2016;18:442-50.

10. Monteiro JM, Ramos MA, Araújo EL, Amorim ELC, Albuquerque UP. Dynamics of medicinal plants knowledge and commerce in an urban ecosystem (Pernambuco, Northeast Brazil). Env Monit Assess. 2011;178:179-202.

11. Lima PGC, Coelho-Ferreira M, Oliveira R. A floresta na feira: plantas medicinais do município de Itaituba, Pará, Brasil. Fragm Cult. 2014;24:285-301.

12. Alves CAB, Silva S, Belarmino NALA, Souza RS, Silva DR, Alves PRR, et al. 
Comercialização de plantas medicinais: um estudo etnobotânico na feira livre do município de Guarabira, Paraíba, Nordeste do Brasil. Gaia Sci. 2016;10:390-407.

13. Mati E, Boer H. Ethnobotany and trade of medicinal plants in the Qaysari Market, Kurdish Autonomous Region, Iraq. J Ethnopharmacol. 2011.133:490-510.

14. Tinitana F, Rios M, Romero-Benavides JC, De La Cruz Rot M, Pardo-De-Santayana M. Medicinal plants sold at traditional markets in southern Ecuador. J Ethnobiol Ethnomed. J Ethnobiol Ethnomed; 2016;12:1-18.

15. Delbanco A-S, Burgess ND, Cuni-Sanchez A. Medicinal Plant Trade in Northern Kenya: Economic Importance, Uses, and Origin. Econ Bot. 2017;71:13-31.

16. Medeiros PM, Haydée Ladio A, Albuquerque UP. Patterns of medicinal plant use by inhabitants of Brazilian urban and rural areas: A macroscale investigation based on available literature. J Ethnopharmacol. 2013;150:729-46.

17. Napagoda MT, Sundarapperuma T, Fonseka D, Amarasiri S, Gunaratna P. Traditional Uses of Medicinal Plants in Polonnaruwa District in North Central Province of Sri Lanka. Scientifica. 2019;2019.

18. Albuquerque UP, Monteiro JM, Ramos MA, Amorim ELC. Medicinal and magic plants from a public market in northeastern Brazil. J Ethnopharmacol. 2007;110:76-91.

19. Brandão MGL, Cosenza GP, Pereira FL, Vasconcelos AS, Fagg CW. Changes in the trade in native medicinal plants in Brazilian public markets. Environ Monit Assess. 2013. 20. Lima PGC, Coelho-Ferreira M, Oliveira R. Plantas medicinais em feiras e mercados públicos do Distrito Florestal Sustentável da BR-163, estado do Pará, Brasil. Acta Bot Bras. $2011 ; 25: 422-34$.

21. Bussmann RW, Paniagua Zambrana NY, Moya Huanca LA, Hart R. Changing markets Medicinal plants in the markets of $\mathrm{La} \mathrm{Paz}$ and El Alto, Bolivia. J Ethnopharmacol. 2016;193:76-95. 
22. Bussmann RW, Sharon D, Vandebroek I, Jones A, Revene Z. Health for sale: the medicinal plant markets in Trujillo and Chiclayo, Northern Peru. J Ethnobiol Ethnomed. $2007 ; 3: 37$.

23. Almeida CFCBR, Albuquerque UP. Uso e conservação de plantas e animais medicinais no estado de pernambuco (nordeste do brasil): Um estudo de caso. Interciencia. 2002;27:27685.

24. Anselmo AF, Silva CG, Marinho M das GV, Zanella FCV, Xavier DA. Levantamento Etnobotânico de Plantas Medicinais Comercializadas por raizeiros em uma Feira Livre no Município de Patos-PB. Biofar. 2012;Especial:39-48.

25. Albuquerque UP, Medeiros PM, Almeida ALS, Monteiro JM, Lins Neto EMF, Melo JG, et al. Medicinal plants of the caatinga (semi-arid) vegetation of NE Brazil: A quantitative approach. J Ethnopharmacol. 2007;114:325-54.

26. Hilonga S, Otieno JN, Ghorbani A, Pereus D, Kocyan A, de Boer H. Trade of wildharvested medicinal plant species in local markets of Tanzania and its implications for conservation. South African J Bot. 2019;122:214-224.

27. Nankaya J, Nampushi J, Petenya S, Balslev H. Ethnomedicinal plants of the Loita Maasai of Kenya. Environ Dev Sustain. 2019;1-21.

28. Catarino S, Duarte MC, Costa E, Carrero PG, Romeiras MM. Conservation and sustainable use of the medicinal Leguminosae plants from Angola. PeerJ . 2019;7:e6736.

29. Maioli-Azevedo V, Fonseca-Kruel VS. Plantas medicinais e ritualísticas vendidas em feiras livres no Município do Rio de Janeiro, RJ, Brasil: estudo de caso nas zonas Norte e Sul RJ, Brazil: a case study in the North and South zones. Acta Bot Brasilica. 2007;21:263-75.

30. Lima PGC, Coelho-Ferreira M, da Silva Santos R. Perspectives on Medicinal Plants in Public Markets across the Amazon: A Review. Econ Bot. 2016;70:64-78.

31. Pala NA, Sarkar BC, Shukla G, Chettri N, Deb S, Bhat JA, et al. Floristic composition and 
utilization of ethnomedicinal plant species in home gardens of the Eastern Himalaya. J Ethnobiol Ethnomed. 2019;15.

32. Panyadee $\mathrm{P}$, Balslev $\mathrm{H}$, Wangpakapattanawong $\mathrm{P}$, Inta A. Medicinal plants in homegardens of four ethnic groups in Thailand. J Ethnopharmacol. 2019;239.

33. Kunwar RM, Mahat L, Acharya RP, Bussmann RW. Medicinal plants, traditional medicine, markets and management in far-west Nepal. J Ethnobiol Ethnomed. 2013;9.

34. Semotiuk AJ, Semotiuk NL, Ezcurra E. The Eruption of Technology in Traditional Medicine: How Social Media Guides the Sale of Natural Plant Products in the Sonoran Desert Region. Econ Bot. 2015;69:360-369.

35. IBGE. IBGE :: Instituto Brasileiro de Geografia e Estatística. 2017.[cited 2018 Sep 13]. https://ww2.ibge.gov.br/home/geociencias/geografia/default_div_int.shtm

36. Moreira ERF. Mesorregiões e microrregiões da Paraiba: delimitação e caracterização. João Pessoa: GAPLAN; 1988.

37. Francisco PRM, Medeiros RM, Santos D, Matos RM. Classificação Climática de Köppen e Thornthwaite para o Estado da Paraíba Paulo. Rev Bras Geogr Física. 2015;8:1006-1016. 38. REFLORA. Flora do Brasil 2020. [cited 2018 Sep 3]. http://floradobrasil.jbrj.gov.br/reflora/listaBrasil/PrincipalUC/PrincipalUC.do\#CondicaoTaxo $\mathrm{nCP}$

39. Tropicos. [cited 2018 Sep 3]. http://www.tropicos.org/

40. ICD. ICD-10 Version:2010 [Internet]. $2010 \quad$ [cited 2018 Sep 26]. http://apps.who.int/classifications/icd10/browse/2010/en

41. Bennett BC, Prance GT. Introduced Plants in the Indigenous Pharmacopoeia of Northern South America. Econ Bot. 2000;54:90-102.

42. Guedes RS, Zanella FCV, Costa Júnior JEV, Santana GM, Silva JA. Caracterização florístico-fitossociológica do componente lenhoso de um trecho de Caatinga no semiárido 
paraibano. Rev. Caatinga. 2012;25:99-108.

43. Sabino FGS, Cunha M do CL, Santana GM. Estrutura da vegetação em dois fragmentos de Caatinga antropizada na Paraíba. Floresta e Ambient. 2016;23:487-97.

44. Yaseen G, Ahmad M, Sultana S, Suleiman Alharrasi A, Hussain J, Zafar M, et al. Ethnobotany of medicinal plants in the Thar Desert (Sindh) of Pakistan. J Ethnopharmacol. 2015;163:43-59.

45. Gomes TB, Bandeira FPS de F. Uso e diversidade de plantas medicinais em uma comunidade quilombola no Raso da Catarina, Bahia. Acta Bot Bras. 2012;26:796-809.

46. Bolson M, Hefler SR, Dall'Oglio Chaves EI, Gasparotto Junior A, Cardozo Junior EL. Ethno-medicinal study of plants used for treatment of human ailments, with residents of the surrounding region of forest fragments of Paraná, Brazil. J Ethnopharmacol. 2015;161:1-10.

47. Albuquerque UP, Oliveira RF. Is the use-impact on native caatinga species in Brazil reduced by the high species richness of medicinal plants? J Ethnopharmacol. 2007;113:156170.

48. Agra MF, Baracho GS, Nurit K, Basílio IJLD, Coelho VPM. Medicinal and poisonous diversity of the flora of "Cariri Paraibano", Brazil. J Ethnopharmacol. 2007;111:383-395.

49. Silva AJR, Andrade LHC. Cultural Significance of Plants in Communities Located in the Coastal Forest Zone of the State of Pernambuco, Brazil. Hum Ecol. 2006;34:447 465.

50. Crepaldi MOS, Peixoto AL. Use and knowledge of plants by "Quilombolas" as subsidies for conservation efforts in an area of Atlantic Forest in Espírito Santo State, Brazil. Biodivers Conserv. 2010;19:37-60.

51. Conde BE, Ticktin T, Fonseca AS, Macedo AL, Orsi TO, Chedier LM, et al. Local ecological knowledge and its relationship with biodiversity conservation among two Quilombola groups living in the Atlantic Rainforest, Brazil. PLoS One. 2017;12:e0187599.

52. Leonti M, Sticher O, Heinrich M. Antiquity of medicinal plant usage in two Macro- 
Mayan ethnic groups (México). J Ethnopharmacol. 2003;88:119-24.

53. Pare D, Hilou A, Ouedraogo N, Guenne S. Ethnobotanical Study of Medicinal Plants Used as Anti-Obesity Remedies in the Nomad and Hunter Communities of Burkina Faso. Medicines. 2016;3:9.

54. Dickel ML, Rates SMK, Ritter MR. Plants popularly used for loosing weight purposes in Porto Alegre, South Brazil. J Ethnopharmacol. 2007;109:60-71.

55. Arenas PM, Molares S, Aguilar Contreras A, Doumecq B, Gabrielli F. Ethnobotanical, micrographic and pharmacological features of plant-based weight-loss products sold in naturist stores in Mexico City: The need for better quality control. Acta Bot Bras. $2013 ; 27: 560-79$.

56. Molares S, Arenas PM, Aguilar A. Etnobotánica urbana de los productos vegetales adelgazantes comercializados en México DF. Bol Latinoam y del Caribe Plantas Med y Aromat. 2012;11:400-412.

57. Cunha MCL, Silva Júnior MC. Flora e Estrutura de Floresta Estacional Semidecidual Montana nos Estados da Paraíba e Pernambuco. Nativa. Revista Nativa; 2014;2:95-102. 
Figure 1. Map of the State of Paraíba, Brazil, highlighting the studied municipalities, the municipalities where it was impossible to conduct the research, the four mesoregions of the state, and the predominance areas of the biomes.

Figure 2. Percentage of citations for each category of medicinal use in public markets of Paraíba, northeastern Brazil. $\mathrm{n}=$ number of informants in the mesoregion. $\mathrm{BCS}=$ Blood and cardiovascular system; CUD = Cultural diseases $;$ DIS = Digestive system $;$ ENM = Endocrine system, nutrition, and metabolism; EXI = External injuries; INT = Intoxication; MBD = Mental and behavioral diseases; MSS = Musculoskeletal system; NEO = Neoplasms; NES = Nervous system; PAD = Parasitic diseases; PRP = Pregnancy and parturition; RES = Respiratory system; RSS = Reproductive system and sexual health; SES = Sensory system; SST $=$ Skin and subcutaneous tissue; UDS = Unspecified diseases and symptoms; URS = Urinary system. A: Mata, B: Agreste, C: Borborema, D: Sertão.

Figure 3. Percentage of citations for the plant parts traded for medicinal use in public markets of Paraíba, northeastern Brazil. $\mathrm{n}=$ number of informants in the Mesoregion. A: Mata, B: Agreste, C: Borborema, D: Sertão.

Figure 4. Principal Coordinates Analysis (PCoA) showing the similarity between the species indicated by the 35 traders in the four mesoregions. Green = Mata; Yellow = Agreste; Blue = Borborema; Red = Sertão. 

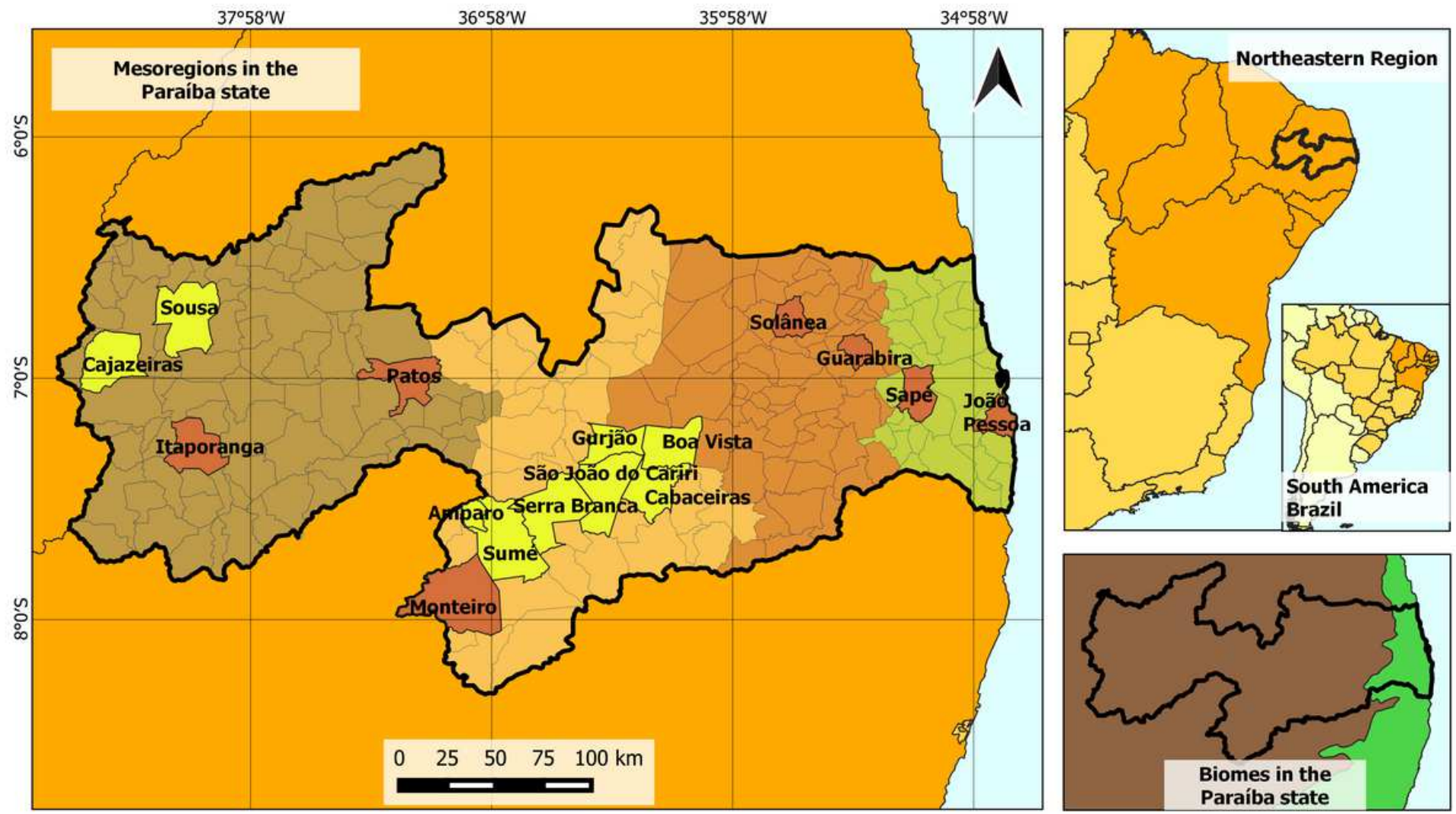

Mata Paraibana

Agreste
Borborema

Sertão
Municipalities where the research was carried out

Municipalities that did not meet the research objectives
Atlantic Forest

Caatinga

\section{Figure 1}

Map of the State of Paraíba, Brazil, highlighting the studied municipalities, the municipalities where it was impossible to conduct the research, the four mesoregions of the state, and the predominance areas of the biomes. 

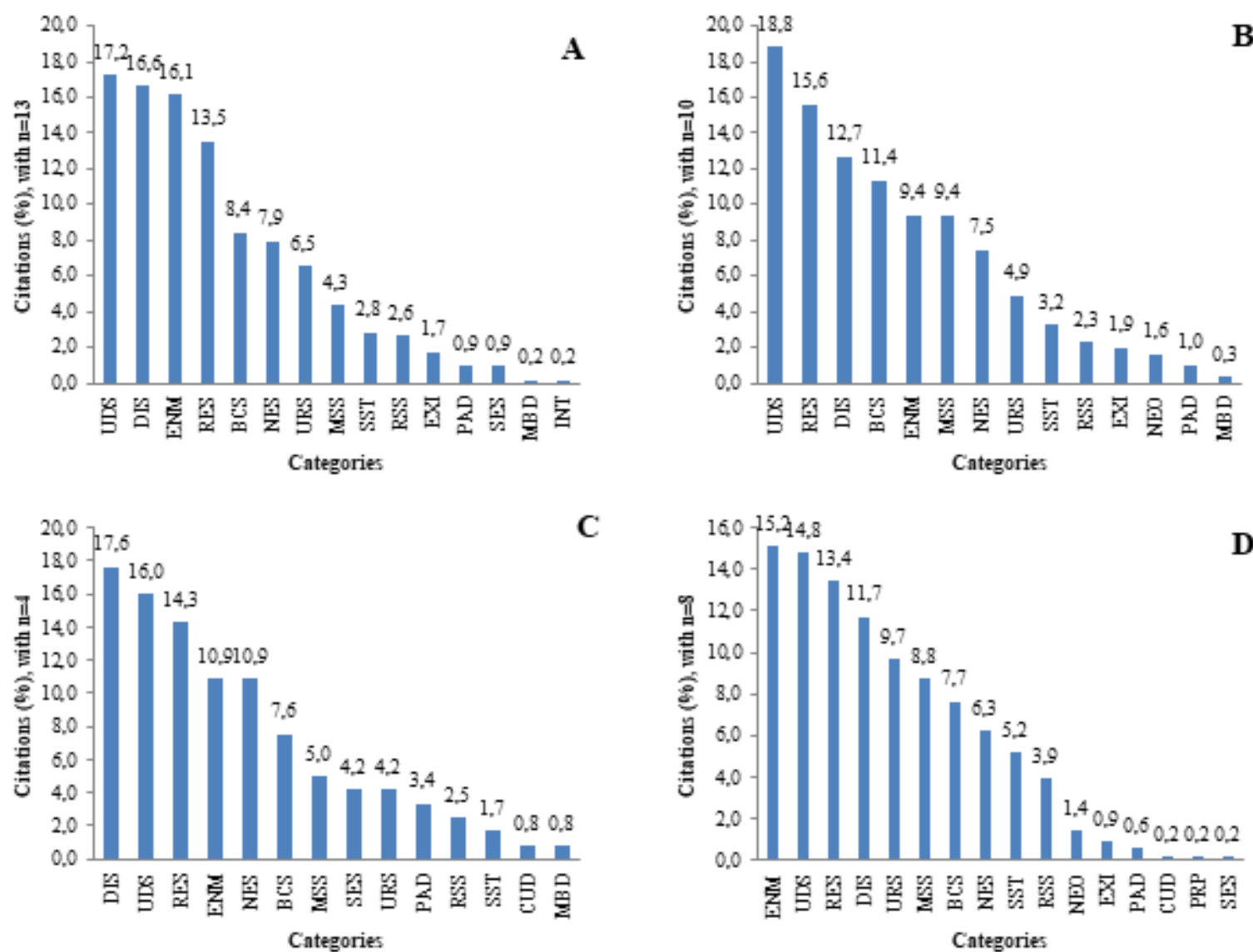

Figure 2

Percentage of citations for each category of medicinal use in public markets of Paraíba, northeastern Brazil. $\mathrm{n}=$ number of informants in the mesoregion. $\mathrm{BCS}=$ Blood and cardiovascular system; $\mathrm{CUD}=$ Cultural diseases; DIS = Digestive system; ENM = Endocrine system, nutrition, and metabolism; EXI = External injuries; INT = Intoxication; MBD = Mental and behavioral diseases; MSS = Musculoskeletal system; NEO = Neoplasms; NES $=$ Nervous system; PAD = Parasitic diseases $;$ PRP $=$ Pregnancy and parturition; RES = Respiratory system; RSS = Reproductive system and sexual health; SES = Sensory system; SST = Skin and subcutaneous tissue; UDS = Unspecified diseases and symptoms; URS = Urinary system. A: Mata, B: Agreste, C: Borborema, D: Sertão. Note: The designations employed and the presentation of the material on this map do not imply the expression of any opinion whatsoever on the part of Research Square concerning the legal status of any country, territory, city or area or of its authorities, or concerning the delimitation of its frontiers or boundaries. This map has been provided by the authors. 


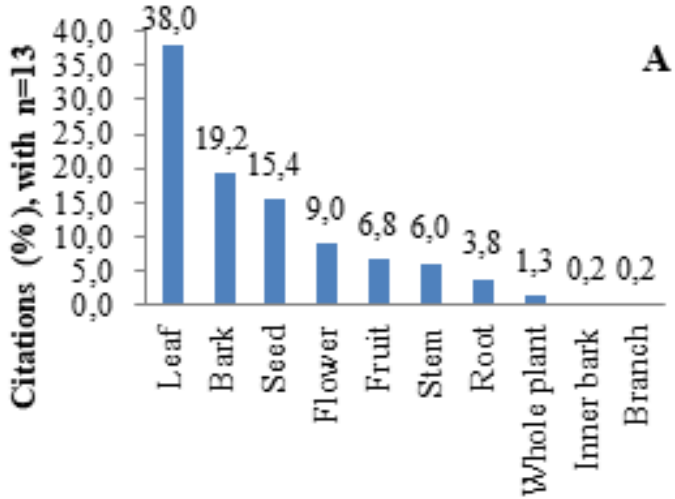

Part used

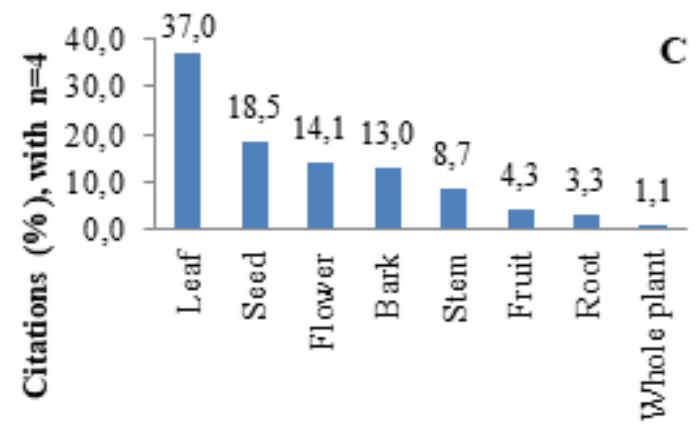

Part used

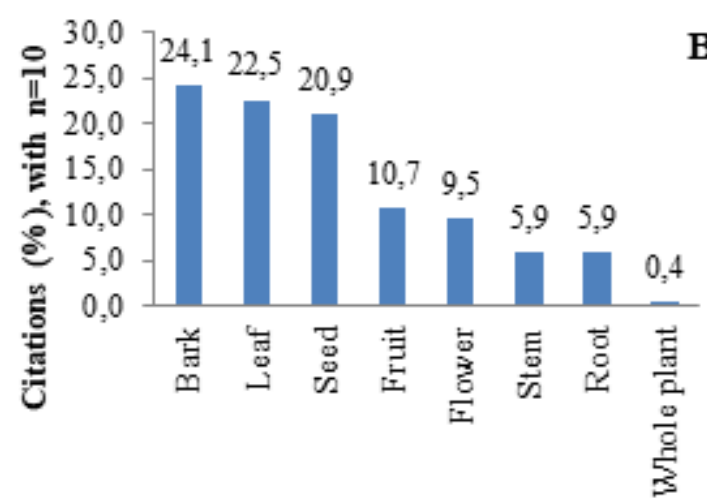

Part used

B

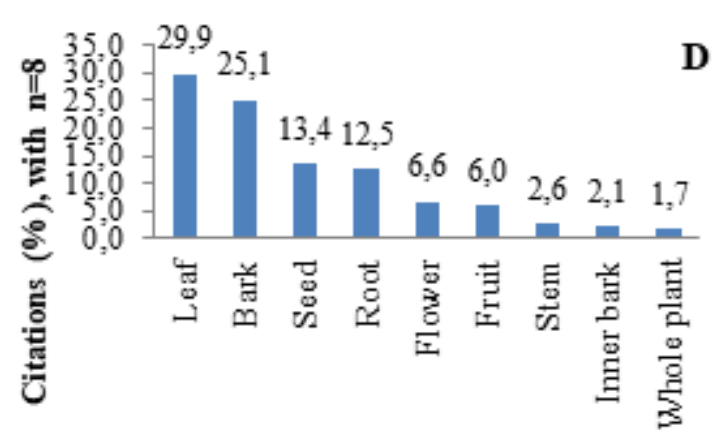

Part used

\section{Figure 3}

Percentage of citations for the plant parts traded for medicinal use in public markets of Paraíba, northeastern Brazil. $\mathrm{n}$ = number of informants in the Mesoregion. A: Mata, B: Agreste, C: Borborema, D: Sertão

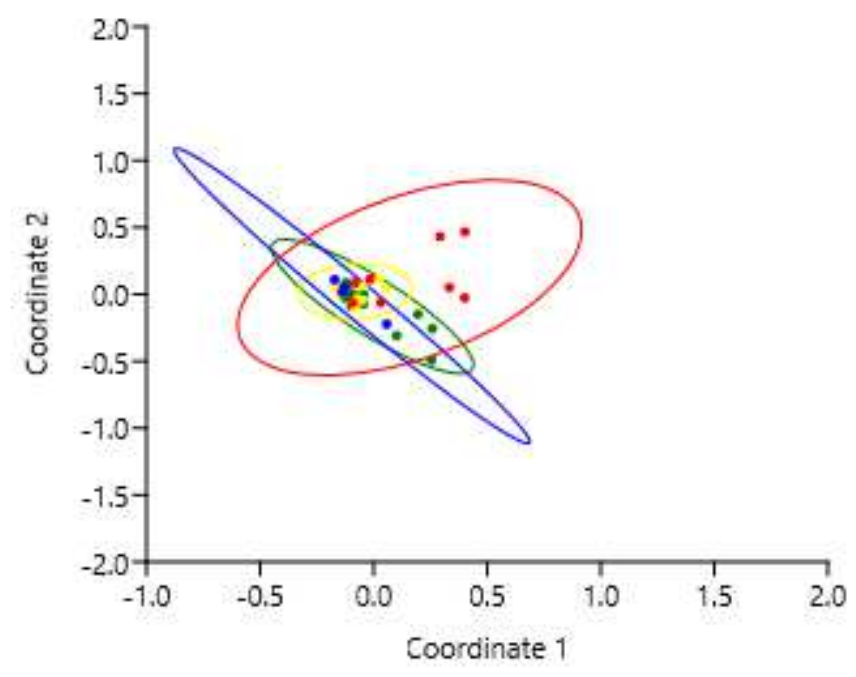

Figure 4

Principal Coordinates Analysis ( $\mathrm{PCOA}$ ) showing the similarity between the species indicated by the 35 traders in the four mesoregions. Green = Mata; Yellow = Agreste; Blue = Borborema; Red $=$ Sertão 